\title{
Dose Delays, Dose Reductions, and Relative Dose Intensity in Patients With Cancer Who Received Adjuvant or Neoadjuvant Chemotherapy in Community Oncology Practices
}

\author{
Neelima Denduluri, MD; ; Debra A. Patt, $\mathrm{MD}^{\mathrm{b}}$; Yunfei Wang, $\mathrm{PhD}^{\mathrm{b}}$; Menaka Bhor, $\mathrm{PhD}^{\mathrm{b}}$; Xiaoyan Li, $\mathrm{PhD}^{\mathrm{c}}$; \\ Anne M. Favret, MD; Phuong Khanh Morrow, MDc; Richard L. Barron, MSc; Lina Asmar, PhD'; \\ Shanmugapriya Saravanan, MS ; Yanli Li, MD, PhDc; Jacob Garcia, MDc; and Gary H. Lyman, MD, MPH
}

\begin{abstract}
Background: A wide variety of myelosuppressive chemotherapy regimens are used for the treatment of cancer in clinical practice. Neutropenic complications, such as febrile neutropenia, are among the most common side effects of chemotherapy, and they often necessitate delays or reductions in doses of myelosuppressive agents. Reduced relative dose intensity (RDI) may lead to poorer disease-free and overall survival. Methods: Using the McKesson Specialty Health/US Oncology iKnowMed electronic health record database, we retrospectively identified the first course of adjuvant or neoadjuvant chemotherapy received by patients without metastases who initiated treatment between January 1, 2007, and March 31, 2011. For each regimen, we estimated the incidences of dose delays ( $\geq 7$ days in any cycle of the course), dose reductions ( $\geq 15 \%$ in any cycle of the course), and reduced RDI ( $<85 \%$ over the course) relative to the corresponding standard tumor regimens described in the NCCN Clinical Practice Guidelines in Oncology (NCCN Guidelines). Results: This study included 16,233 patients with 6 different tumor types who received 1 of 20 chemotherapy regimens. Chemotherapy dose delays, dose reductions, and reduced RDI were common among patients treated in community oncology practices in the United States, but RDI was highly variable across patients, regimens, and tumor types (0.486-0.935 for standard tumor regimen cohorts). Reduced RDI was more common in older patients, obese patients, and patients whose daily activities were restricted. Conclusions: In this large evaluation of RDI in US clinical practice, physicians frequently administered myelosuppressive agents at dose intensities lower than those of standard regimens. (J Natl Compr Canc Netw 2015;13:1383-1393)
\end{abstract}

\section{Background}

The NCCN Clinical Practice Guidelines in Oncology (NCCN Guidelines) recommend a wide variety of myelosuppressive chemotherapy regimens for the treatment of cancer in adjuvant and neoadjuvant settings. ${ }^{1-7}$ Neutropenia is one of the leading dose-limiting toxicities of chemotherapy, and neutropenic complications,

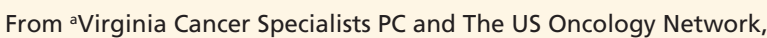
Arlington, Virginia; ' ${ }^{b}$ McKesson Specialty Health and The US Oncology Network, The Woodlands, Texas; 'Amgen Inc., Thousand Oaks, California; dVirginia Cancer Specialists PC and The US Oncology Network, Fairfax,

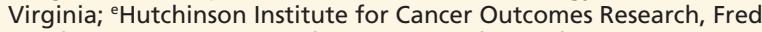
Hutchinson Cancer Research Center, Seattle, Washington.

Submitted December 16, 2014; accepted for publication

August 18, 2015.

Xiaoyan Li, Phuong Khanh Morrow, Richard L Barron, Yanli Li, and Jacob Garcia are employed by and own stock in Amgen Inc. Gary H. Lyman is the especially chemotherapy-induced febrile neutropenia (FN), often lead physicians to reduce or delay planned doses of chemotherapy so as to limit myelotoxicity and allow recovery of neutrophil counts. ${ }^{8-11}$

Accumulating clinical evidence suggests that optimal outcomes are achieved with standard chemotherapy regimens, and chemotherapy dose delays and dose reductions result in poorer disease-free, progression-free,

principal investigator of a research grant to the Fred Hutchinson Cancer Research Center from Amgen. The remaining authors have disclosed that they have no financial interests, arrangements, affiliations, or commercial interests with the manufacturers of any products discussed in this article or their competitors.

This study was funded by Amgen Inc.

Correspondence: Gary H. Lyman, MD, MPH, 1100 Fairview Avenue North, M3-B232, PO Box 19024, Seattle, WA 98109-1024.

E-mail: glyman@fhcrc.org 
Denduluri et al

and overall survivals among patients with curable malignancies receiving adjuvant and neoadjuvant therapies. ${ }^{8,10,12-21}$ An emphasis has therefore been placed on maintaining full-dose chemotherapy on the planned schedule in clinical practice as a means to improve patient outcomes. ${ }^{22}$

Relative dose intensity (RDI), or the ratio of delivered dose intensity to standard or planned dose intensity, is a summary measure that is commonly used to describe dose delays and/or reductions that occur within a chemotherapy course. ${ }^{20,23}$ An RDI less than $85 \%$ is generally considered to be a clinically significant reduction from standard or planned therapy. ${ }^{8,10,12,20,24}$ Prophylactic granulocyte colonystimulating factor (G-CSF) use has been shown in randomized controlled trials and observational studies to reduce the risk of reduced RDI. 8,10,17,25,26

In this retrospective cohort study, we estimated the incidence of chemotherapy dose delays, dose reductions, missing doses, and reduced RDI relative to standard chemotherapy regimens among 16,233 patients with 6 tumor types who were treated with adjuvant or neoadjuvant chemotherapy regimens currently in widespread use in community oncology practices in the United States.

\section{Methods}

\section{Data Source and Study Design}

The McKesson Specialty Health/US Oncology iKnowMed Electronic Health Record (EHR) database captures outpatient practice encounter history for patients treated by more than 1000 oncologists and hematologists in nearly 230 McKesson Specialty Health/US Oncology practices that are geographically distributed across the United States. iKnowMed captures demographic information, laboratory values, diagnoses, planned and actual therapy administration, line of therapy, stage at diagnosis, comorbidities, and performance status. Oncologists enter planned chemotherapy agents, planned cycle length, and planned number of cycles into coded data fields in the database before chemotherapy initiation.

Using the iKnowMed EHR database, we retrospectively identified the first course of adjuvant or neoadjuvant chemotherapy received by patients with nonmetastatic cancer and assigned patients to specific tumor chemotherapy regimen cohorts (see supplementary eTable 1) based on chemotherapy agents received in cycle 1 , planned number of cycles, and planned duration of each cycle for all 6 tumor types. We then compared the treatment received by patients in each cohort with standard tumor regimens as described in the NCCN Guidelines. ${ }^{1-7}$

\section{Study Population}

The study included patients aged 18 years or older treated for stage I-IIIA female breast cancer, stage IIII ovarian cancer, stage I-IIIA non-small cell lung cancer (NSCLC), stage I-III Hodgkin lymphoma, stage I-III non-Hodgkin's lymphoma (NHL), or stage I-III colorectal cancer (CRC). All patients initiated adjuvant or neoadjuvant chemotherapy at McKesson Specialty Health/US Oncology sites using the full EHR capacities of the iKnowMed database between January 1, 2007, and March 31, 2011.

Patients included in the study received at least one intravenously administered myelosuppressive agent in the first chemotherapy cycle, had no evidence of distant metastasis before chemotherapy initiation, did not receive any oral myelosuppressive agents during the chemotherapy course, were not treated for another tumor type, and did not participate in any clinical trials during the chemotherapy course.

Adjuvant or neoadjuvant chemotherapy was determined based on the value of the "line of therapy" variable in the database: "adjuvant" or "neoadjuvant" for patients with breast cancer, ovarian cancer, NSCLC, or CRC; "first line" for patients with NHL or Hodgkin lymphoma.

Only standard tumor regimen cohorts consisting of 100 or more patients were included in this study. This cohort size was chosen so as to achieve a precision level (anticipated half width of 95\% CIs) for the primary end point ( $\%$ of patients with reduced RDI) of approximately $10 \%$ or less. Based on this precision level, we calculated the required minimum sample size for each regimen to be approximately 100 .

\section{Outcome Measures}

A dose delay was identified if a patient experienced a delay of 7 or more days in the administration of at least 1 myelosuppressive agent in any chemotherapy cycle relative to the standard day of administration. $8,10,22,27,28$

A dose reduction was identified if a patient experienced a reduction of $15 \%$ or more in chemotherapy dose for at least 1 myelosuppressive agent 
in any chemotherapy cycle relative to the standard dose. ${ }^{8,10,22,27,28}$

A missing dose was identified if a patient did not receive at least 1 myelosuppressive agent that was part of the standard regimen in any cycle. A patient with a missing dose in a cycle was assumed to have both a dose delay and a dose reduction in that cycle. This same approach to missing doses was used in previous studies. ${ }^{8,10,22,27,28}$

RDI was defined as the ratio of delivered dose intensity to the dose intensity of the standard tumor regimen (standard dose, standard cycle length, and standard number of cycles as described in the NCCN Guidelines). Delivered dose intensity was defined as total delivered dose divided by actual time to complete chemotherapy or standard time to complete chemotherapy, whichever was longer. RDI was measured for each myelosuppressive agent in a regimen over the entire chemotherapy course and then averaged across all of the myelosuppressive agents in a regimen. An RDI less than $85 \%$ was designated as the clinically meaningful threshold for reduction in RDI based on previously published studies. ${ }^{8,10,14,17,19,20,22,27,28}$

\section{Statistical Analyses}

For each standard tumor regimen cohort, RDI was calculated during the first chemotherapy course, and dose delays and dose reductions were identified in any cycle of the course. Descriptive statistics were reported by standard tumor regimen cohort for patient baseline characteristics (measured at chemotherapy initiation), incidence of dose delays ( $\geq 7$ days) in any cycle of the course, incidence of dose reductions $(\geq 15 \%)$ in any cycle of the course, percentage of patients with any missing dose for any myelosuppressive agent, RDI (as a continuous variable), and incidence of reduced RDI $(<85 \%)$ over the course. Similar analyses of incidence of reduced RDI were conducted on subgroups of patients based on patient and disease characteristics.

Sensitivity analyses were conducted using planned regimen (planned dose, planned cycle length, and planned number of cycles) as the benchmark for calculation of dose delays, dose reductions, and reduced RDI. Planned dose for a myelosuppressive agent was assumed to be the maximum of all doses received across cycles (which was the first administered dose for almost all patients included in the study). For patients with lymphoma and CRC, an alternative "hybrid" benchmark for RDI less than $85 \%$ was also used that included dose and cycle length from the standard regimen and number of cycles from the planned regimen. This hybrid benchmark was used because the NCCN Guidelines give physicians flexibility when determining the appropriate number of chemotherapy cycles. Sensitivity analyses were also conducted by varying the threshold for defining reduced RDI $(<90 \%,<95 \%)$ or dose reduction $(\geq 5 \%, \geq 10 \%)$.

Patients with evidence of disease progression, unplanned regimen change, or death were censored beyond the first event date.

\section{Results}

\section{Characteristics of the Study Population}

This study included 16,233 patients with 6 different tumor types who received 1 of 20 chemotherapy regimens. Characteristics of the study population by standard regimen cohort and regimen definitions and abbreviations are shown in Table 1.

\section{Dose Delays, Dose Reductions, and RDI}

As shown in Figure 1, a considerable proportion of patients across regimens had dose delays (22.9\%$88.4 \%)$ and dose reductions $(22.3 \%-93.1 \%)$. A considerable proportion of patients $(14.7 \%-87.6 \%)$ also missed at least 1 dose of a myelosuppressive agent that is part of the standard regimen. As shown in Figure 2, RDI less than $85 \%$ ranged from $15.6 \%$ for patients with breast cancer treated with dose-dense AC followed by paclitaxel every 2 weeks to $87.6 \%$ for patients with CRC treated with 5 -FU (every 8 weeks).

Colony-stimulating factor (CSF) prophylaxis in cycle 1 was consistently high for the 3 breast cancer regimens with a high risk ( $>20 \%$ ) of $\mathrm{FN}$ according to the NCCN Guidelines for Myeloid Growth Factors $^{29}$ (from $89.1 \%$ for dose-dense AC followed by paclitaxel every 2 weeks to $92.1 \%$ for dose-dense AC followed by paclitaxel every week; Table 2) (for the most recent version of these guidelines, visit NCCN. org). The mean RDI was high in these regimens (0.857-0.935; Table 2), and RDI less than $85 \%$ was low (15.6\%-27.1\%; Figure 2). CSF prophylaxis in cycle 1 was much more variable for regimens with an intermediate risk (10\%-20\%) of FN according to the NCCN Guidelines for Myeloid Growth Factors ${ }^{29}$ 
Denduluri et al

\begin{tabular}{|c|c|c|c|c|c|c|c|c|c|c|c|c|c|c|c|c|c|c|c|c|}
\hline \multirow{2}{*}{$\overrightarrow{\underline{x}}$} & $\mathrm{~d} / \mathrm{d} / \mathrm{d} / \mathrm{d}$ & g & 命 & & : & 今ે & 㝏 & $\mid \begin{array}{l}\infty \\
\dot{\sigma} \\
\dot{\sigma}\end{array}$ & ố & $\stackrel{\substack{n \\
m}}{m}$ & & $\begin{array}{l}0 \\
\dot{y}\end{array}$ & 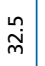 & 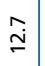 & $\stackrel{؟}{=}$ & 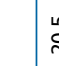 & 盀 & 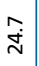 & 貼 & \multirow{2}{*}{ 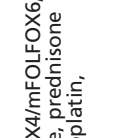 } \\
\hline & $\begin{array}{r}(y M \varepsilon b) \text { dOHJ } \\
/ d O H J-y\end{array}$ & fo & 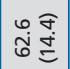 & & $\stackrel{N}{\simeq}$ & $\underset{\substack{n \\
m}}{n}$ & 离 & $\frac{\dot{q}}{\bar{N}}$ & $\stackrel{n}{\sim}$ & î & & ğ & \begin{tabular}{ll}
\multirow{2}{*}{} \\
$\mathrm{m}$
\end{tabular} & ใู & ळ. & & 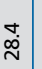 & 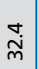 & ֻू & \\
\hline 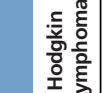 & 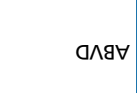 & $\stackrel{\Sigma}{2}$ & 离鬲 & & స్రి & ğ & $\begin{array}{l}\llcorner \\
\infty \\
\infty\end{array}$ & हैं & 䍦 & 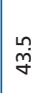 & & $\overline{\mathrm{U}}$ & 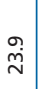 & $\stackrel{\circ}{m}$ & $\stackrel{m}{\rho}$ & & 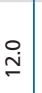 & ָ̃. & 㐫 & 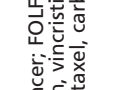 \\
\hline & $(x \rightarrow 8 b) n-s$ & \& & 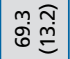 & & $\stackrel{\circ}{\circ}$ & 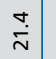 & 昜 & $\bar{f}$ & 字 & 文 & & $\begin{array}{l}0 \\
\stackrel{0}{\circ} \\
\bullet\end{array}$ & $\underset{\tilde{m}}{\mathbb{m}}$ & $\bar{j}$ & : & & $\hat{o}$ & $\begin{array}{ll}n \\
m \\
m\end{array}$ & $\begin{array}{l}\infty \\
\dot{0} \\
\dot{0}\end{array}$ & 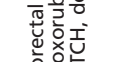 \\
\hline שֶّ & 9X0-7าองس & 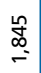 & 胫 & & $\stackrel{\circ}{\circ}$ & $\stackrel{m}{\ddagger}$ & $\begin{array}{l}\stackrel{N}{N} \\
\end{array}$ & $\stackrel{n}{2}$ & 绝 & 㞷 & & 官 & $\stackrel{\sim}{m}$ & : & $\stackrel{n}{0}$ & & ó & $\begin{array}{l}\tilde{\infty} \\
\stackrel{\infty}{ }\end{array}$ & $\overline{\dot{\infty}}$ & \\
\hline & tXOAาOA & $\stackrel{\circ}{n}$ & 竞 & & f & $\begin{array}{l}0 \\
\dot{q}\end{array}$ & $\stackrel{\stackrel{\sim}{i}}{i}$ & $\stackrel{\unlhd}{\beth}$ & 㓊 & $\stackrel{N}{\tilde{m}}$ & & $\underset{N}{N}$ & $\overline{\dot{m}}$ & : & $\stackrel{m}{ \pm}$ & & $\stackrel{?}{\stackrel{n}{-}}$ & 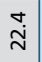 & $\bar{i}$ & 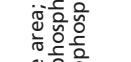 \\
\hline U & 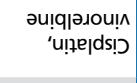 & $\stackrel{\infty}{\sim}$ & 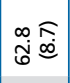 & & $\stackrel{n}{n}$ & $\stackrel{\infty}{\stackrel{\infty}{n}}$ & 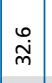 & $\overline{\dot{0}}$ & 望 & $\stackrel{\substack{n \\
\sim}}{\sim}$ & & :̊. & $\begin{array}{l}\infty \\
\infty \\
\infty\end{array}$ & $\stackrel{8}{\circ}$ & $\stackrel{n}{=}$ & & 市 & 离 & \begin{tabular}{l}
\multirow{2}{0}{} \\
$\stackrel{m}{m}$
\end{tabular} & \\
\hline$\frac{n}{z}$ & $\begin{array}{l}\text { Irxę|ped } \\
\text { 'ụpepdoques }\end{array}$ & $\bar{m}$ & 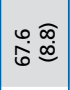 & & $\stackrel{m}{m}$ & ָे & $\mid \begin{array}{l}\infty \\
\dot{f} \\
\end{array}$ & $\stackrel{\infty}{\stackrel{\sim}{\sim}}$ & 它 & 吕 & & $\overline{\overline{6}}$ & 今̊ & $\stackrel{\infty}{-}$ & 이 & & ָ̃ & $\begin{array}{l}M \\
\stackrel{m}{0} \\
m\end{array}$ & 吕 & \\
\hline 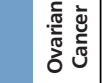 & 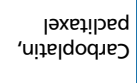 & $\frac{t}{m}$ & 官 & & $\stackrel{\mathbb{N}}{\stackrel{5}{=}}$ & $\begin{array}{l}\dot{q} \\
\dot{y}\end{array}$ & 望 & $\stackrel{\stackrel{n}{\prime}}{=}$ & $\frac{\hat{s}}{z}$ & $\stackrel{\infty}{\infty}$ & & $\stackrel{m}{n}$ & $\stackrel{m}{q}$ & $\therefore$ & 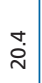 & & 家 & $\hat{i}$ & $\begin{array}{l}\dot{\sigma} \\
\dot{\sigma}\end{array}$ & \\
\hline & $\begin{array}{r}\text { әххегәวор } \\
\leftarrow \supset \forall\end{array}$ & זे & 官命 & & 虽 & ָี & $\stackrel{\sim}{\stackrel{N}{I}}$ & $\hat{m}$ & $\stackrel{a}{z}$ & iे & & $\bar{g}$ & กี & $\stackrel{\infty}{\circ}$ & $\underset{\dot{I}}{q}$ & & 葛 & ó & ฉे & \\
\hline & 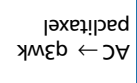 & 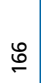 & 苫全 & & $\overline{\grave{N}}$ & $\begin{array}{l}+ \\
\dot{\sigma}\end{array}$ & \begin{tabular}{|l|}
$-\bar{\infty}$ \\
$\infty$
\end{tabular} & î & $\mathbf{s}$ & $\stackrel{m}{\sim}$ & & ֻू & F & $\therefore$ & $\mid \begin{array}{l}0 \\
\stackrel{0}{0}\end{array}$ & & $\stackrel{n}{=}$ & ì. & $\stackrel{\infty}{\infty}$ & \\
\hline & 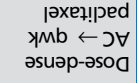 & $\underset{m}{\infty}$ & $\begin{array}{l}\text { 经 } \\
\text { in }\end{array}$ & & $\widehat{o b}$ & $\stackrel{\check{\gamma}}{\check{\gamma}}$ & 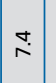 & $\stackrel{\leftrightarrow}{-}$ & $\frac{1}{z}$ & $\stackrel{n}{n}$ & & $\begin{array}{l}\infty \\
\dot{0} \\
\infty\end{array}$ & 古 & : & 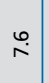 & & ڤ్ & 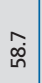 & $\stackrel{\substack{d \\
d}}{d}$ & \\
\hline & & 号 & 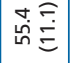 & & 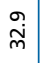 & $\overline{\dot{g}}$ & $\stackrel{I}{i}$ & $\stackrel{m}{q}$ & $\stackrel{n}{z}$ & $\stackrel{\infty}{\sim}$ & & 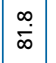 & $\stackrel{\infty}{\infty}$ & m & कू & & $\bar{\infty} \underset{\infty}{\sim}$ & 诂 & $\stackrel{\mathfrak{N}}{\stackrel{1}{*}}$ & \\
\hline & $\underset{\text { әзиәр-әsос }}{\supset}$ & 宗 & 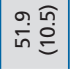 & & $\stackrel{\leftrightarrow}{\mathscr{\gamma}}$ & \begin{tabular}{l|}
0 \\
$\dot{\gamma}$
\end{tabular} & $\overline{\underline{I}}$ & $\cong$ & $\stackrel{q}{z}$ & $\begin{array}{l}m \\
g \\
g\end{array}$ & & \begin{tabular}{l}
\multicolumn{1}{c}{$\mid$} \\
$\infty$ \\
$\infty$
\end{tabular} & in & $:$ & $\stackrel{\infty}{\infty}$ & & 点 & กี่ & $\stackrel{m}{\sim}$ & \\
\hline 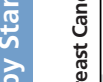 & 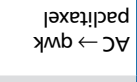 & $\bar{\gamma}$ & 象商 & & $\overline{\dot{m}}$ & $\underset{\ddagger}{\ddagger}$ & $\mid \begin{array}{l}a \\
\dot{g}\end{array}$ & 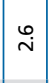 & $\stackrel{\mathbb{s}}{z}$ & $\check{I}$ & & $\begin{array}{l}\sigma \\
\dot{b} \\
\infty\end{array}$ & $\stackrel{\circ}{m}$ & $\tilde{o}$ & $\begin{array}{l}n \\
\infty \\
\sigma\end{array}$ & & 兽 & 임 & $\stackrel{\substack{q \\
\dot{q}}}{\sim}$ & \\
\hline & HOI & $\stackrel{\substack{m \\
=}}{=}$ & 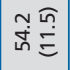 & & $\stackrel{n}{\stackrel{n}{m}}$ & $\begin{array}{l}0 \\
\dot{g}\end{array}$ & $\begin{array}{l}\dot{a} \\
\dot{m}\end{array}$ & $\hat{f}$ & $\frac{\tilde{s}}{z}$ & 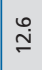 & & 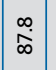 & $\bar{\sigma}$ & $\overline{0}$ & $\infty$ & & $\begin{array}{l}\text { 它 } \\
\dot{m}\end{array}$ & $\stackrel{n}{\bar{n}}$ & $\stackrel{9}{=}$ & \\
\hline & נב (9-כאקן) & $\stackrel{?}{g}$ & in & & $\stackrel{\sim}{\sim}$ & $\frac{q}{y}$ & $\hat{i}$ & กิ & $\frac{\pi}{z}$ & $\bar{i}$ & & $\begin{array}{l}\infty \\
\stackrel{\infty}{j} \\
\infty\end{array}$ & 8 & İ & $\stackrel{\infty}{0}$ & & $\underset{\sim}{\stackrel{\Delta}{\sim}}$ & $\stackrel{\simeq}{\square}$ & $\stackrel{n}{=}$ & \\
\hline & 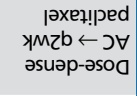 & $\stackrel{8}{\stackrel{\sigma}{\sigma}}$ & 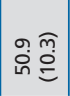 & & 每 & $\hat{f}$ & \%ू & $\hat{o}$ & $\frac{5}{z}$ & $\stackrel{\infty}{\stackrel{\infty}{\sim}}$ & & $\begin{array}{c}0 \\
\infty \\
\infty\end{array}$ & $\tilde{m}$ & $\overline{0}$ & $\stackrel{9}{r}$ & & $\stackrel{P}{\dot{f}}$ & 要 & 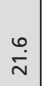 & \\
\hline & $\supset \forall 1$ & $\begin{array}{l}\text { : } \\
\stackrel{\circ}{n}\end{array}$ & 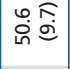 & & $\begin{array}{l}\stackrel{g}{\sigma} \\
\dot{\sigma}\end{array}$ & $\hat{\dot{y}}$ & \begin{tabular}{l|}
0 \\
$\infty$
\end{tabular} & $\begin{array}{l}\infty \\
0 \\
0\end{array}$ & $\stackrel{\pi}{z}_{z}^{\infty}$ & $\begin{array}{l}\infty \\
\infty \\
\infty \\
\sim\end{array}$ & & $\begin{array}{l}0 \\
\infty \\
\infty \\
\infty \\
\infty\end{array}$ & $\stackrel{\sim}{\mathrm{N}}$ & $\overline{0}$ & $\hat{\infty}$ & & $\begin{array}{l}\stackrel{0}{\circ} \\
\stackrel{0}{*}\end{array}$ & $\mid \begin{array}{l}m \\
f \\
0\end{array}$ & $\overline{\dot{\sim}}$ & \\
\hline & בד (ק-t) & బ్ల్ల & in & & 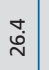 & 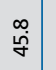 & $\stackrel{\grave{\sim}}{\grave{N}}$ & $\overline{6}$ & $\frac{\$}{z}$ & $\begin{array}{l}0 \\
\stackrel{0}{\infty} \\
\stackrel{\infty}{*}\end{array}$ & & $\begin{array}{c}a \\
\dot{\infty} \\
\infty\end{array}$ & $\dot{q}$ & $\begin{array}{l}n \\
0\end{array}$ & హ. & & 范 & 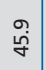 & $\hat{m}$ & \\
\hline & & $z$ & 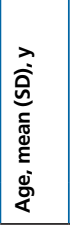 & 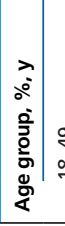 & $\underset{\substack{g \\
\infty}}{ } \mid$ & $\begin{array}{l}0 \\
\vdots \\
0 \\
0\end{array} \mid$ & $\left|\begin{array}{l}0 \\
1 \\
0 \\
0\end{array}\right|$ & $\stackrel{n}{n}$ & 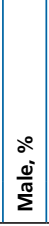 & 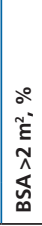 & 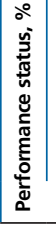 & 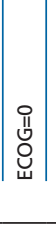 & $\left|\begin{array}{|l}\mid \pi \\
0 \\
⿱ \\
ن\end{array}\right|$ & $\mid$\begin{tabular}{|c}
$\mid$ \\
$\tilde{O}$ \\
$\tilde{U}$ \\
\end{tabular} & $\begin{array}{l}\text { s. } \\
\text { o. } \\
\frac{5}{5}\end{array}$ & 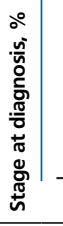 & & & $\supseteqq$ & 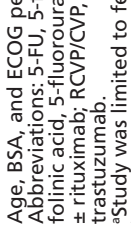 \\
\hline
\end{tabular}

(from $1.4 \%$ for NSCLC: cisplatin, vinorelbine, to $73.1 \%$ for NHL: R-CHOP/CHOP [every 3 weeks]; Table 2). The mean RDI was likewise quite variable (0.546-0.898; Table 2) as was RDI less than 85\% (19.3\%-82.1\%; Figure 2). Patients who received intermediate-risk regimens constituted most of the patients in the study $(n=9,626)$ and contributed the most to the variation in RDI.

The mean RDI ranged from 0.486 to 0.935 , and was highest in the breast cancer regimens (Table 2). The median RDI ranged from 0.451 to 0.982 , and was also highest in the breast cancer regimens. 


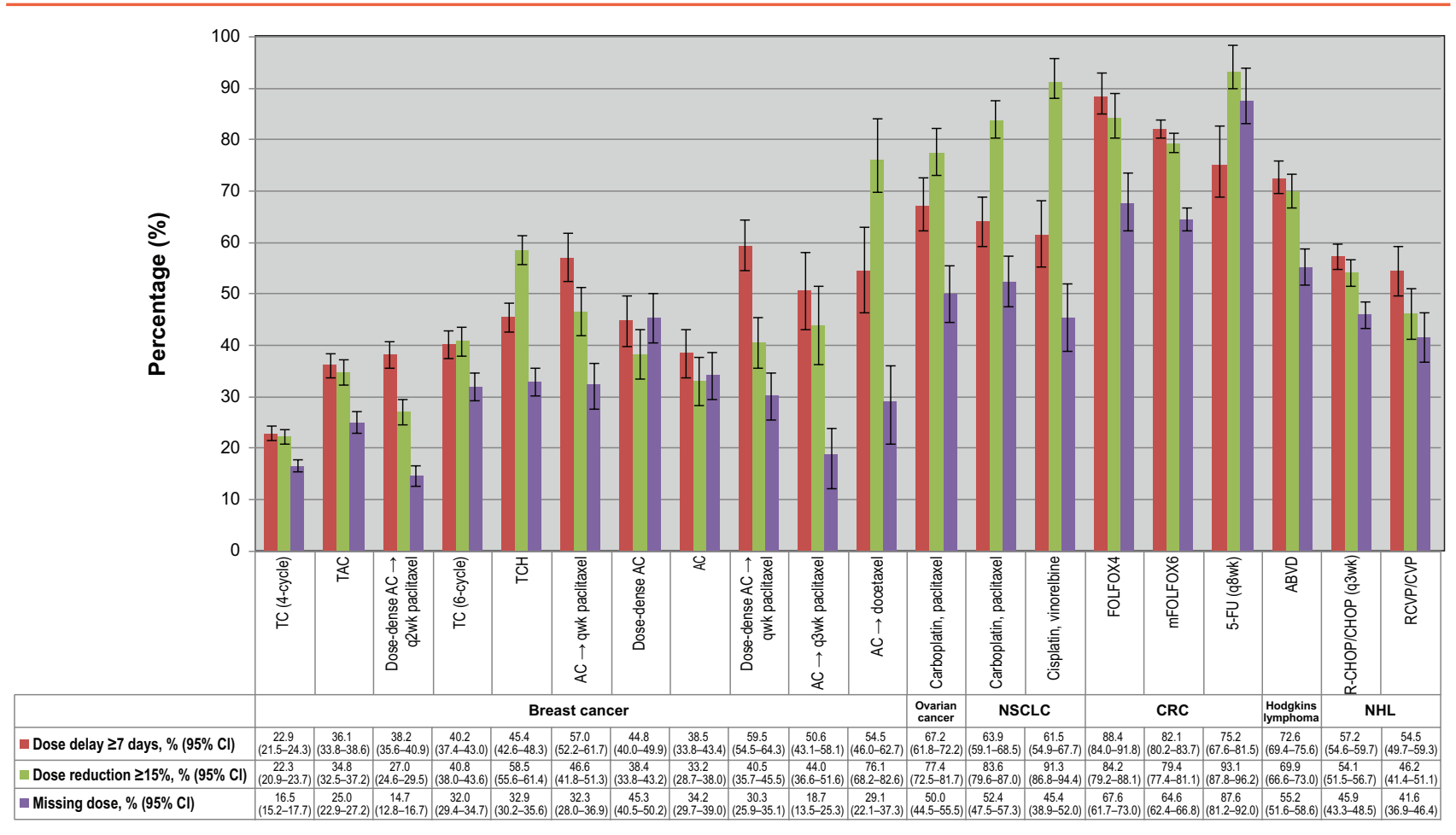

Standard Regimen Cohort

Figure 1 Incidence of dose delays, dose reductions, and missing doses. Missing doses were based on percentage of patients with any missing dose for any myelosuppressive agent. Error bars indicate $95 \% \mathrm{Cl}$.

Abbreviations: 5-FU, 5-fluorouracil; ABVD, doxorubicin, bleomycin, vinblastine, dacarbazine; AC, doxorubicin, cyclophosphamide; CRC, colorectal cancer; FOLFOX4/mFOLFOX6, folinic acid, 5-fluorouracil, oxaliplatin; NHL, non-Hodgkin lymphoma; NSCLC, non-small cell lung cancer; R-CHOP/CHOP, cyclophosphamide, doxorubicin, vincristine, prednisone \pm rituximab; RCVP/CVP, cyclophosphamide, vincristine, prednisone \pm rituximab; RDI, relative dose intensity; TAC, docetaxel, doxorubicin, cyclophosphamide; TC, docetaxel, cyclophosphamide; TCH, docetaxel, carboplatin, trastuzumab.

Perhaps most significantly, RDI varied considerably across patients within tumor regimen cohorts (Table 2). The 25 th percentile for RDI was as low as 0.210 and as high as 0.904 , whereas the 75 th percentile for RDI was as low as 0.713 and as high as 0.999 . This wide variation within standard tumor regimen cohorts is apparent in histograms that show the distribution of RDI (supplementary eFigure 1).

For most tumor types, the incidences of reduced RDI were higher in older patients (age $\geq 65$ years), in obese patients (body surface area $[\mathrm{BSA}]>2 \mathrm{~m}^{2}$ ), and in patients whose daily activities were restricted (ECOG performance status $\geq 1$ ) (Table 3 ). The percentage of patients who received reduced RDI $(<85 \%)$ generally increased with age among those with breast cancer, CRC, Hodgkin lymphoma, and NHL.

Sensitivity analyses showed that estimates for the incidence of reduced RDI and dose reduction were higher when more restrictive alternative thresholds were used (Table 4). Sensitivity analyses also showed that estimates for the incidence of dose delays, dose reductions, and reduced RDI were generally lower when a planned regimen was used as the benchmark (Table 4).

\section{Limitations}

The results presented herein reflect the patient population and treatment patterns for McKesson Specialty Health/US Oncology practices. Services, medications, and procedures provided to patients outside the McKesson Specialty Health/US Oncology Network were not captured by the iKnowMed EHR database and could not be ascertained in this study. This study relied on coded data fields in the iKnowMed database rather than on data extracted from medical charts; this limited our ability to comprehensively measure certain clinical characteristics (eg, comorbidities) and understand rationales for dose delays and reductions. Treatment history before a patient's first encounter at a US Oncology practice may only be available in physician progress notes 


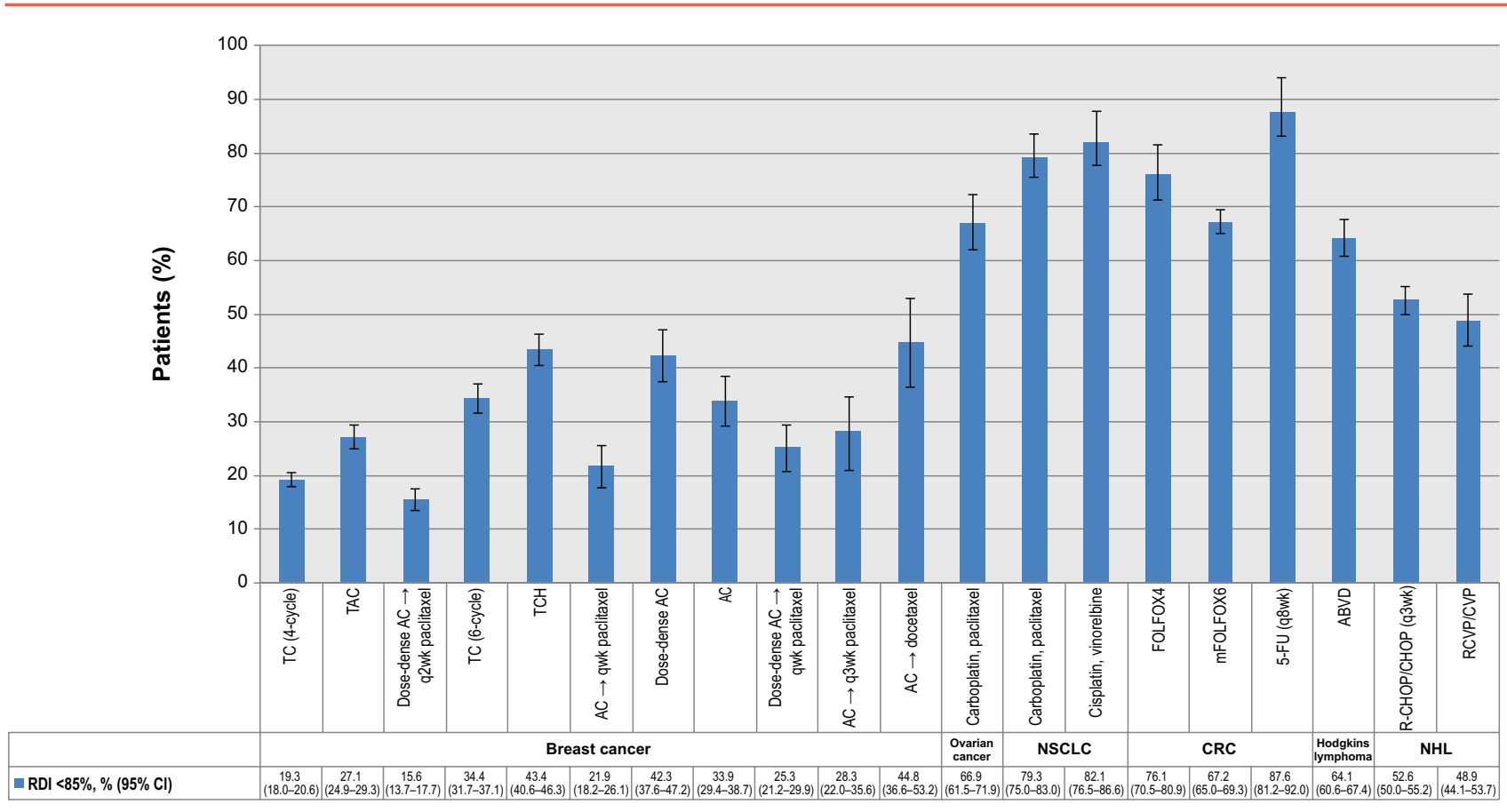

Standard Regimen Cohort

Figure 2 Incidence of reduced RDI. Error bars indicate $95 \% \mathrm{Cl}$.

Abbreviations: 5-FU, 5-fluorouracil; ABVD, doxorubicin, bleomycin, vinblastine, dacarbazine; AC, doxorubicin, cyclophosphamide; CRC, colorectal cancer; FOLFOX4/mFOLFOX6, folinic acid, 5-fluorouracil, oxaliplatin; NHL, non-Hodgkin lymphoma; NSCLC, non-small cell lung cancer; R-CHOP/CHOP, cyclophosphamide, doxorubicin, vincristine, prednisone \pm rituximab; RCVP/CVP, cyclophosphamide, vincristine, prednisone \pm rituximab; RDI, relative dose intensity; TAC, docetaxel, doxorubicin, cyclophosphamide; TC, docetaxel, cyclophosphamide; TCH, docetaxel, carboplatin, trastuzumab.

and is not well captured in the structured elements of the iKnowMed EHR. As with all administrative databases, the data in the iKnowMed database are subject to coding errors of omission and commission. Some patient characteristics were missing or unknown (eg, performance status); no attempt was made to impute missing values. For some tumor types and chemotherapy regimens, a dose range is listed in the NCCN Guidelines. In these cases, a single dose (listed in supplementary eTable 1) was selected as the standard dose based on the scientific literature. This may have impacted the degree of reduced RDI and dose reduction observed in this study.

\section{Discussion}

Chemotherapy dose delays, dose reductions, and reduced RDI were common among patients treated in community oncology practices in the United States, but RDI was highly variable across patients, regimens, and tumor types. Incidences of dose delays, dose reductions, missing doses, and reduced RDI were lowest among patients with breast cancer and highest in those with CRC. Incidences of all of these measures exceeded $40 \%$ in NHL, $45 \%$ in NSCLC, and 55\% in Hodgkin lymphoma.

Patients with breast cancer were generally younger than those with other cancer types except Hodgkin lymphoma. Young age may have contributed to the low incidence of reduced RDI, because physicians may have believed that the patients could cope with the dose intensity prescribed by standard regimens. ${ }^{30,31}$ Physicians may also have believed that the prognosis for patients with breast cancer was relatively good, and therefore may have administered full dose intensity as a result. ${ }^{22}$ Some of the most common regimens used to treat breast cancer incorporated primary prophylaxis with growth factors in their pivotal trials (eg, dosedense AC-T). ${ }^{32}$ Practitioners therefore had a systematic guide to follow that included relatively aggressive treatment with adequate supportive care.

Patients with advanced age, high BSA, or poor performance status were at higher risk of receiving reduced RDI. In the case of older patients and patients with ECOG performance status of 1 or higher, physicians may have been cautious in their dosing out of concern for excessive toxicity. ${ }^{33,34}$ This concern may have inadvertently led to undertreat- 


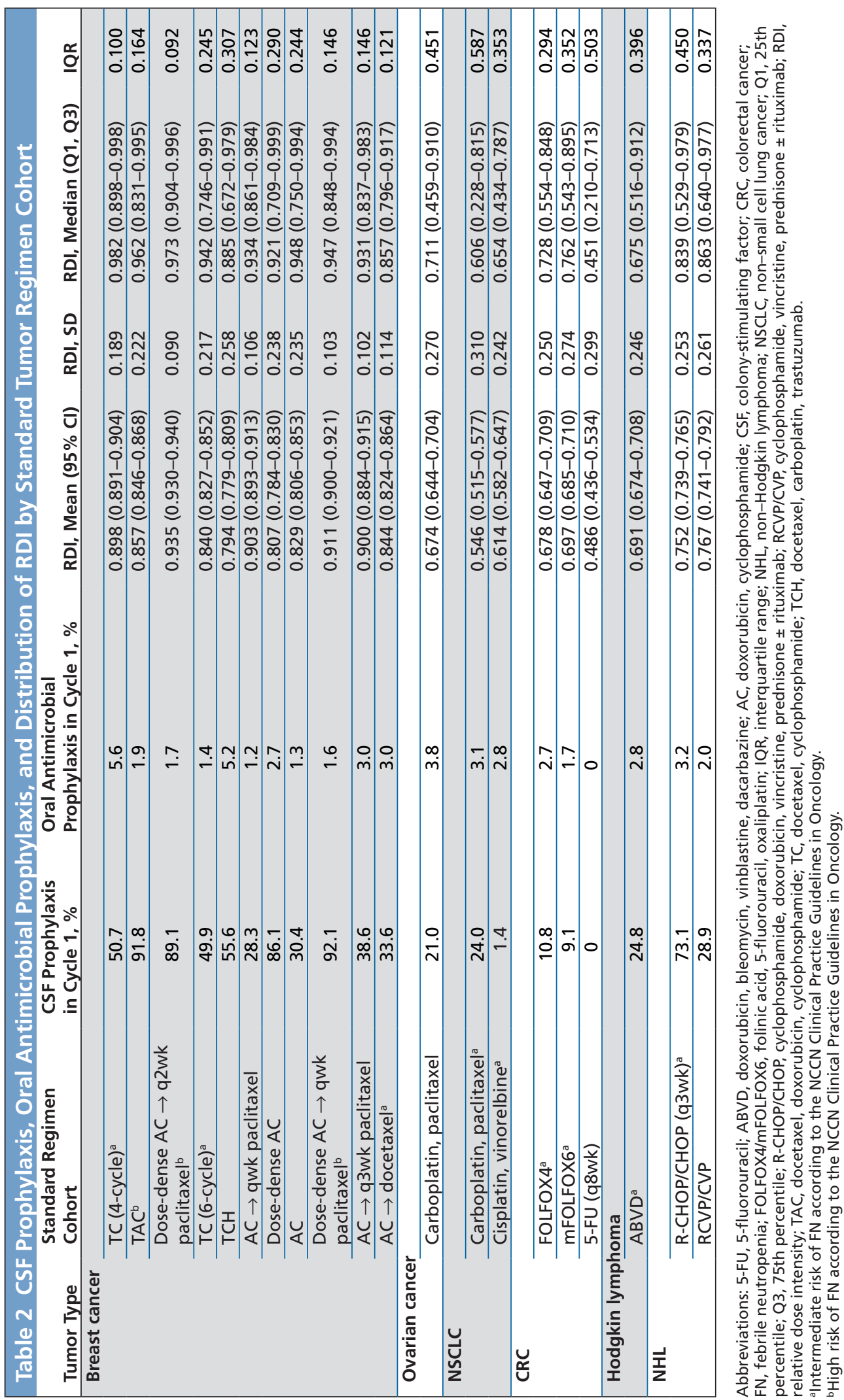


Denduluri et al

\begin{tabular}{|c|c|c|c|c|c|c|}
\hline \multirow{2}{*}{$\begin{array}{l}\text { Patient Characteristics } \\
\text { Overall, } \%(95 \% \mathrm{Cl})\end{array}$} & $\begin{array}{l}\text { Breast Cancer } \\
(\mathrm{N}=10,435)\end{array}$ & $\begin{array}{c}\text { Ovarian Cancer } \\
(\mathrm{N}=314)\end{array}$ & $\begin{array}{c}\text { NSCLC } \\
(\mathrm{N}=609)\end{array}$ & $\begin{array}{c}\text { CRC } \\
(\mathrm{N}=2,249)\end{array}$ & $\begin{array}{l}\text { Hodgkin } \\
\text { Lymphoma } \\
(\mathrm{N}=774)\end{array}$ & $\begin{array}{c}\mathrm{NHL} \\
(\mathrm{N}=1,852)\end{array}$ \\
\hline & $\begin{array}{c}26.6 \\
(25.7-27.4)\end{array}$ & $\begin{array}{c}66.9 \\
(61.5-71.9) \\
\end{array}$ & $\begin{array}{c}80.3 \\
(77.0-83.3)\end{array}$ & $\begin{array}{c}69.5 \\
(67.6-71.4)\end{array}$ & $\begin{array}{c}64.1 \\
(60.6-67.4)\end{array}$ & $\begin{array}{c}51.8 \\
(49.5-54.1)\end{array}$ \\
\hline \multicolumn{7}{|l|}{ Age group, \% $(95 \% \mathrm{Cl})$} \\
\hline $18-49 y$ & $\begin{array}{c}23.5 \\
(22.1-24.9)\end{array}$ & $\begin{array}{c}68.5 \\
(55.3-79.3)\end{array}$ & Not shown ${ }^{b}$ & $\begin{array}{c}63.7 \\
(58.9-68.2)\end{array}$ & $\begin{array}{c}62.3 \\
(58.0-66.4)\end{array}$ & $\begin{array}{c}43.3 \\
(37.5-49.2)\end{array}$ \\
\hline $50-64$ y & $\begin{array}{c}25.7 \\
(24.7-26.9) \\
\end{array}$ & $\begin{array}{c}66.7 \\
(58.6-73.8) \\
\end{array}$ & $\begin{array}{c}78.9 \\
(73.1-83.7) \\
\end{array}$ & $\begin{array}{c}66.5 \\
(63.5-69.4) \\
\end{array}$ & $\begin{array}{c}63.0 \\
(55.1-70.2) \\
\end{array}$ & $\begin{array}{c}45.8 \\
(41.8-49.9) \\
\end{array}$ \\
\hline $65-74$ y & $\begin{array}{c}32.0 \\
(29.7-34.2)\end{array}$ & $\begin{array}{c}60.0 \\
(49.0-70.0)\end{array}$ & $\begin{array}{c}81.7 \\
(76.4-86.0)\end{array}$ & $\begin{array}{c}71.1 \\
(67.3-74.5)\end{array}$ & $\begin{array}{c}71.2 \\
(59.4-80.7)\end{array}$ & $\begin{array}{c}47.5 \\
(43.2-51.8)\end{array}$ \\
\hline $75+y$ & $\begin{array}{c}41.6 \\
(36.8-46.5)\end{array}$ & $\begin{array}{c}80.6 \\
(65.0-90.2)\end{array}$ & $\begin{array}{c}80.2 \\
(71.8-86.5)\end{array}$ & $\begin{array}{c}85.7 \\
(81.0-89.3)\end{array}$ & $\begin{array}{c}78.6 \\
(64.1-88.3) \\
\end{array}$ & $\begin{array}{c}68.2 \\
(63.9-72.2) \\
\end{array}$ \\
\hline \multicolumn{7}{|l|}{ Sex, \% $(95 \% \mathrm{Cl})$} \\
\hline Female & $\begin{array}{c}26.6 \\
(25.7-27.4)\end{array}$ & $\begin{array}{c}66.9 \\
(61.5-71.9)\end{array}$ & $\begin{array}{c}78.2 \\
(73.1-82.6)\end{array}$ & $\begin{array}{c}74.8 \\
(72.0-77.3)\end{array}$ & $\begin{array}{c}61.7 \\
(56.6-66.5)\end{array}$ & $\begin{array}{c}52.7 \\
(49.5-56.0)\end{array}$ \\
\hline Male & N/A & N/A & $\begin{array}{c}82.1 \\
(77.6-85.9)\end{array}$ & $\begin{array}{c}65.2 \\
(62.5-67.8)\end{array}$ & $\begin{array}{c}66.3 \\
(61.6-70.8) \\
\end{array}$ & $\begin{array}{c}50.9 \\
(47.7-54.0)\end{array}$ \\
\hline \multicolumn{7}{|l|}{ BSA, \% (95\% Cl) } \\
\hline$\leq 2 \mathrm{~m}^{2}$ & $\begin{array}{c}25.4 \\
(24.5-26.3)\end{array}$ & $\begin{array}{c}64.2 \\
(58.2-69.8)\end{array}$ & $\begin{array}{c}77.3 \\
(73.2-81.0)\end{array}$ & $\begin{array}{c}69.3 \\
(66.8-71.6)\end{array}$ & $\begin{array}{c}62.9 \\
(58.3-67.3)\end{array}$ & $\begin{array}{c}51.7 \\
(48.9-54.6)\end{array}$ \\
\hline$>2 \mathrm{~m}^{2}$ & $\begin{array}{c}42.2 \\
(37.8-46.7)\end{array}$ & $\begin{array}{c}78.9 \\
(66.7-87.5)\end{array}$ & $\begin{array}{c}87.8 \\
(82.1-91.9)\end{array}$ & $\begin{array}{c}70.0 \\
(66.8-73.0)\end{array}$ & $\begin{array}{c}65.6 \\
(60.4-70.5)\end{array}$ & $\begin{array}{c}51.9 \\
(48.1-55.6)\end{array}$ \\
\hline \multicolumn{7}{|c|}{ Performance status, \% $(95 \% \mathrm{Cl})$} \\
\hline$E C O G=0$ & $\begin{array}{c}24.5 \\
(23.5-25.4)\end{array}$ & $\begin{array}{c}66.3 \\
(59.6-72.5)\end{array}$ & $\begin{array}{c}77.7 \\
(73.4-81.6)\end{array}$ & $\begin{array}{c}67.6 \\
(65.3-69.8)\end{array}$ & $\begin{array}{c}65.1 \\
(60.7-69.2)\end{array}$ & $\begin{array}{c}48.2 \\
(44.9-51.5)\end{array}$ \\
\hline $\mathrm{ECOG} \geq 1$ & $\begin{array}{c}35.8 \\
(33.6-38.0)\end{array}$ & $\begin{array}{c}73.3 \\
(59.0-84.0)\end{array}$ & $\begin{array}{c}84.9 \\
(78.7-89.6)\end{array}$ & $\begin{array}{c}76.2 \\
(71.4-80.4)\end{array}$ & $\begin{array}{c}60.6 \\
(53.9-66.9)\end{array}$ & $\begin{array}{c}55.1 \\
(51.6-58.6)\end{array}$ \\
\hline \multicolumn{7}{|c|}{ Stage at diagnosis, \% $(95 \% \mathrm{Cl})$} \\
\hline 1 & $\begin{array}{c}25.1 \\
(23.6-26.6)\end{array}$ & $\begin{array}{c}73.0 \\
(64.5-80.0)\end{array}$ & $\begin{array}{c}76.6 \\
(69.3-82.6)\end{array}$ & Not shown ${ }^{b}$ & $\begin{array}{c}88.2 \\
(80.1-93.3)\end{array}$ & $\begin{array}{c}68.2 \\
(63.9-72.1)\end{array}$ \\
\hline II & $\begin{array}{c}26.6 \\
(25.5-27.8)\end{array}$ & $\begin{array}{c}64.6 \\
(52.5-75.1)\end{array}$ & $\begin{array}{c}79.3 \\
(73.7-83.9)\end{array}$ & $\begin{array}{c}71.8 \\
(67.4-75.8)\end{array}$ & $\begin{array}{c}65.5 \\
(60.9-69.7)\end{array}$ & $\begin{array}{c}48.3 \\
(44.2-52.4)\end{array}$ \\
\hline III & $\begin{array}{c}29.3 \\
(27.0-31.7)\end{array}$ & $\begin{array}{c}62.2 \\
(53.5-70.2)\end{array}$ & $\begin{array}{c}84.1 \\
(78.6-88.4)\end{array}$ & $\begin{array}{c}68.7 \\
(66.5-70.8)\end{array}$ & $\begin{array}{c}52.1 \\
(45.8-58.4)\end{array}$ & $\begin{array}{c}44.1 \\
(40.6-47.5)\end{array}$ \\
\hline
\end{tabular}

Abbreviations: BSA, body surface area; CRC, colorectal cancer; N/A, not applicable; NHL, non-Hodgkin's lymphoma; NSCLC, non-small cell lung cancer; RDI, relative dose intensity.

a Data are presented as the percentage $(95 \% \mathrm{Cl})$ of patients in each subgroup with reduced RDI.

${ }^{\mathrm{b}}$ Not shown because sample size was $<30$.

ment. ${ }^{35,36}$ Physicians may have dosed obese patients to ideal body weight or may have top-coded BSA values at $2 \mathrm{~m}^{2}$, rather than using actual weight and actual BSA values. This was common practice before the 2012 release of the ASCO guideline on appropriate chemotherapy dosing of obese patients, ${ }^{37,38}$ because methodology in some randomized clinical trials mandated capping BSA at $2 \mathrm{~m}^{2}$.

In patients with some tumor types, dose reductions of $15 \%$ or greater and reduced RDI were less common when planned regimens were used for comparison than when standard regimens were used (Table 4). This suggests that some physicians planned reductions in RDI (eg, with planned dose reductions or planned number of cycles lower than standard) before actual chemotherapy initiation. In other words, physicians may have reduced the dose intensity both in anticipation of and in response to toxicity. Similar planned reductions in dose and RDI have been observed in patients with $\mathrm{NHL}^{8,10}$ and breast cancer, ${ }^{39}$ and in patients aged 65 years or older with solid tumors. ${ }^{40,41}$

Neutropenia and FN are the most common toxicity-related reason for dose delays and reductions among patients with breast cancer, ${ }^{22} \mathrm{NHL},{ }^{27}$ and NSCLC. ${ }^{42}$ Other hematologic toxicities, including anemia, ${ }^{43}$ thrombocytopenia, ${ }^{44}$ and nonhematologic toxicities, such as fatigue, ${ }^{45,46}$ nausea and vomiting, ${ }^{47}$ neuropathy, ${ }^{42,48}$ mucositis, ${ }^{49}$ renal dysfunction, ${ }^{20}$ patient request, and weight change, ${ }^{22,27}$ may also lead to dose delays and reductions.

Deviations from standard regimens as a result of toxicity might potentially be reduced by increasing 
Chemotherapy Relative Dose Intensity

\begin{tabular}{|c|c|c|c|c|c|c|}
\hline & $\begin{array}{c}\text { Breast } \\
\text { Cancer } \\
(\mathrm{N}=10,435)\end{array}$ & $\begin{array}{l}\text { Ovarian } \\
\text { Cancer } \\
(\mathrm{N}=314)\end{array}$ & $\begin{array}{l}\text { NSCLC } \\
(\mathrm{N}=609)\end{array}$ & $\begin{array}{c}\text { CRC } \\
(\mathrm{N}=2,249)\end{array}$ & $\begin{array}{l}\text { Hodgkin } \\
\text { Lymphoma } \\
(\mathrm{N}=774)\end{array}$ & $\begin{array}{c}\mathrm{NHL} \\
(\mathrm{N}=1,852)\end{array}$ \\
\hline $\begin{array}{l}\text { Dose delay based on } \geq 7 \text { days and standard } \\
\text { regimen (base case estimate), } \%(95 \% \mathrm{Cl})\end{array}$ & $\begin{array}{c}36.1 \\
(35.2-37.1)\end{array}$ & $\begin{array}{c}67.2 \\
(61.8-72.2)\end{array}$ & $\begin{array}{c}63.1 \\
(59.2-66.8)\end{array}$ & $\begin{array}{c}82.3 \\
(80.7-83.9)\end{array}$ & $\begin{array}{c}72.6 \\
(69.4-75.6)\end{array}$ & $\begin{array}{c}56.6 \\
(54.3-58.8)\end{array}$ \\
\hline $\begin{array}{l}\text { Dose delay } \geq 7 \text { days based on planned } \\
\text { regimen, } \%(95 \% \mathrm{Cl})\end{array}$ & $\begin{array}{c}38.0 \\
(37.1-39.0)\end{array}$ & $\begin{array}{c}61.5 \\
(56.0-66.7)\end{array}$ & $\begin{array}{c}65.7 \\
(61.8-69.3)\end{array}$ & $\begin{array}{c}80.5 \\
(78.8-82.1)\end{array}$ & $\begin{array}{c}66.9 \\
(63.5-70.1)\end{array}$ & $\begin{array}{c}63.7 \\
(61.4-65.8)\end{array}$ \\
\hline $\begin{array}{l}\text { Dose reduction based on } \geq 15 \% \text { and standard } \\
\text { regimen (base case estimate), } \%(95 \% \mathrm{Cl})\end{array}$ & $\begin{array}{c}34.5 \\
(33.6-35.4)\end{array}$ & $\begin{array}{c}77.4 \\
(72.4-81.7)\end{array}$ & $\begin{array}{c}82.9 \\
(79.7-85.7)\end{array}$ & $\begin{array}{c}80.8 \\
(79.1-82.4)\end{array}$ & $\begin{array}{c}69.9 \\
(66.6-73.0)\end{array}$ & $\begin{array}{c}52.4 \\
(50.1-54.6)\end{array}$ \\
\hline $\begin{array}{l}\text { Threshold for dose reduction changed } \\
\text { to } 10 \% \text { (based on standard regimen), } \\
\%(95 \% \mathrm{Cl})\end{array}$ & $\begin{array}{c}40.1 \\
(39.1-41.0)\end{array}$ & $\begin{array}{c}79.9 \\
(75.2-84.0)\end{array}$ & $\begin{array}{c}92.9 \\
(90.6-94.7)\end{array}$ & $\begin{array}{c}83.8 \\
(82.2-85.2)\end{array}$ & $\begin{array}{c}72.6 \\
(69.4-75.6)\end{array}$ & $\begin{array}{c}56.8 \\
(54.5-59.0)\end{array}$ \\
\hline $\begin{array}{l}\text { Threshold for dose reduction changed } \\
\text { to } 5 \% \text { (based on standard regimen), } \\
\%(95 \% \mathrm{Cl})\end{array}$ & $\begin{array}{c}44.9 \\
(44.0-45.9)\end{array}$ & $\begin{array}{c}83.1 \\
(78.6-86.9)\end{array}$ & $\begin{array}{c}93.8 \\
(91.6-95.4)\end{array}$ & $\begin{array}{c}87.6 \\
(86.2-88.9)\end{array}$ & $\begin{array}{c}79.1 \\
(76.1-81.8)\end{array}$ & $\begin{array}{c}61.8 \\
(59.6-64.0)\end{array}$ \\
\hline $\begin{array}{l}\text { Dose reduction } \geq 15 \% \text { based on } \\
\text { planned regimen, } \%(95 \% \mathrm{Cl})\end{array}$ & $\begin{array}{c}32.7 \\
(31.8-33.6)\end{array}$ & $\begin{array}{c}58.0 \\
(52.4-63.3)\end{array}$ & $\begin{array}{c}67.8 \\
(64.0-71.4)\end{array}$ & $\begin{array}{c}77.8 \\
(76.0-79.4)\end{array}$ & $\begin{array}{c}59.0 \\
(55.5-62.5)\end{array}$ & $\begin{array}{c}55.7 \\
(53.5-58.0)\end{array}$ \\
\hline $\begin{array}{l}\text { Reduced RDI based on }<85 \% \text { and standard } \\
\text { regimen (base case estimate), } \%(95 \% \mathrm{Cl})\end{array}$ & $\begin{array}{c}26.6 \\
(25.7-27.4)\end{array}$ & $\begin{array}{c}66.9 \\
(61.5-71.9)\end{array}$ & $\begin{array}{c}75.2 \\
(71.6-78.5)\end{array}$ & $\begin{array}{c}69.5 \\
(67.6-71.4)\end{array}$ & $\begin{array}{c}64.1 \\
(60.6-67.4)\end{array}$ & $\begin{array}{c}51.8 \\
(49.5-54.1)\end{array}$ \\
\hline $\begin{array}{l}\text { Threshold for reduced RDI changed to } \\
90 \% \text { (based on standard regimen), } \\
\%(95 \% \mathrm{Cl})\end{array}$ & $\begin{array}{c}34.2 \\
(33.3-35.1)\end{array}$ & $\begin{array}{c}73.3 \\
(68.1-77.8)\end{array}$ & $\begin{array}{c}84.9 \\
(81.8-87.5)\end{array}$ & $\begin{array}{c}77.9 \\
(76.1-79.6)\end{array}$ & $\begin{array}{c}72.1 \\
(68.8-75.1)\end{array}$ & $\begin{array}{c}58.4 \\
(56.2-60.6)\end{array}$ \\
\hline $\begin{array}{l}\text { Threshold for reduced RDI changed to } \\
95 \% \text { (based on standard regimen), } \\
\%(95 \% \mathrm{Cl})\end{array}$ & $\begin{array}{c}46.5 \\
(45.6-47.5)\end{array}$ & $\begin{array}{c}80.3 \\
(75.5-84.3)\end{array}$ & $\begin{array}{c}90.0 \\
(87.3-92.1)\end{array}$ & $\begin{array}{c}86.5 \\
(85.0-87.8)\end{array}$ & $\begin{array}{c}83.2 \\
(80.4-85.7)\end{array}$ & $\begin{array}{c}68.0 \\
(65.8-70.1)\end{array}$ \\
\hline $\begin{array}{l}\text { RDI }<85 \% \text { based on standard dose and } \\
\text { cycle length and planned number of } \\
\text { cycles, } \%(95 \% \mathrm{Cl})^{\text {a }}\end{array}$ & & & & $\begin{array}{c}66.9 \\
(64.9-68.8)\end{array}$ & $\begin{array}{c}55.9 \\
(52.4-59.4)\end{array}$ & $\begin{array}{c}51.0 \\
(48.7-53.2)\end{array}$ \\
\hline $\begin{array}{l}\mathrm{RDI}<85 \% \text { based on planned regimen, } \\
\%(95 \% \mathrm{Cl})\end{array}$ & $\begin{array}{c}27.3 \\
(26.5-28.2)\end{array}$ & $\begin{array}{c}50.0 \\
(44.5-55.5)\end{array}$ & $\begin{array}{c}62.2 \\
(58.3-66.0)\end{array}$ & $\begin{array}{c}67.4 \\
(65.4-69.3)\end{array}$ & $\begin{array}{c}52.8 \\
(49.3-56.3)\end{array}$ & $\begin{array}{c}54.4 \\
(52.1-56.6)\end{array}$ \\
\hline
\end{tabular}

Abbreviations: CRC, colorectal cancer; NHL, non-Hodgkin's lymphoma; NSCLC, non-small cell lung cancer; RDI, relative dose intensity.

${ }^{a} \mathrm{RDI}<85 \%$ based on standard dose and cycle length and planned number of cycles was calculated for patients with lymphoma and CRC because NCCN Guidelines give physicians flexibility when determining the appropriate number of chemotherapy cycles.

awareness among physicians of the importance of maintaining RDI and providing adequate supportive care. Physicians used primary G-CSF prophylaxis more frequently with regimens documented as having a high risk of FN in the NCCN Guidelines (eg, TAC, dose-dense AC-T). Incidences of dose delays, dose reductions, and reduced RDI for TAC and dose-dense AC followed by paclitaxel, regimens that are listed in the NCCN Guidelines as having a high risk (>20\%) of FN, ${ }^{1,29}$ were not substantially higher than those of other regimens (Figures 1 and 2). Rates of CSF primary prophylaxis were $89 \%$ to $92 \%$ in patients who received these regimens (Table 2). Other factors, such as younger age and better performance status, could also contribute to a higher RDI in some patient populations.

In clinical practice, many patients received chemotherapy at dose intensities that were lower than those in standard regimens. Increased communication between physicians and patients regarding the survival benefits of using standard regimens and preserving RDI might improve adherence to prescribed chemotherapy dose and schedule. However, many physicians may also have well-justified reasons to delay or reduce doses of myelosuppressive agents (eg, for elderly patients and patients with serious comorbidities), and these delays and reductions may be planned before chemotherapy initiation. ${ }^{8,10}$ The reasons behind decisions by physicians to reduce or delay doses of myelosuppressive agents were not captured in the coded data fields of the iKnowMed database and were not analyzed in this study.

The standard regimens specified in the NCCN Guidelines are based on clinical trial protocols. 
Denduluri et al

Some patient groups who are not well represented in clinical trials (eg, older or obese patients) may fare poorly with standard regimens. In this study, patients with CRC who received 5-FU (every 8 weeks) were older than patients in many other cohorts (Table 1 ), and the prevalence of dose delays, dose reductions, missing doses, and reduced RDI were all very high (Figures 1 and 2). Although the NCCN Guidelines recommend dose adjustments based on individual patient risk factors, ${ }^{29}$ patients from underrepresented populations should ideally be included in clinical trials so as to better establish doses that show both reasonable toxicity and efficacy.

The findings of this study are qualitatively similar to those of other studies. ${ }^{9,22,27,28,50}$ Lyman et $\mathrm{al}^{22,27}$ and Weycker et $\mathrm{al}^{28}$ reported recent estimates for the incidence of reduced RDI among patients with early-stage breast cancer and NHL. Culakova et $\mathrm{al}^{50}$ reported incidence of reduced RDI in patients with solid tumors or lymphoma, and included patients with small cell lung cancer and those with stage IV disease (who were not captured in our study). Compared with corresponding estimates in previous studies with similar calculation algorithms for dose delays, dose reductions, and reduced RDI, 8,10 more recent estimates are relatively lower, despite an increase in the use of taxane-based regimens that may be more myelotoxic. ${ }^{22,51}$ In 2003, Lyman et $\mathrm{al}^{8}$ reported reduced RDI in $56 \%$ of patients with earlystage breast cancer, whereas in the present study, the corresponding estimate was $27 \%$. The publication of clinical practice guidelines by $\mathrm{NCCN}^{1-7,29}$ and by $\mathrm{ASCO}^{37,38}$; increased physician awareness of the benefits of maintaining high RDI; and increased GCSF use may all have contributed to this increase in RDI.

\section{Conclusions}

This study provides current estimates for the incidence of reduced RDI among patients with earlystage breast cancer, NHL, NSCLC, ovarian cancer, $\mathrm{CRC}$, and Hodgkin lymphoma treated with chemotherapy regimens that are in widespread use in clinical practice. This study also captures "real world" practice patterns over a wide range of geographic regions treated in both rural and urban settings. Further research should evaluate the impact of RDI on longterm patient outcomes (eg, overall survival) among the patients in this study. Future research should also examine the reasons behind physicians' decisions to reduce or delay doses of myelosuppressive agents. This might require a survey of physicians, because reasons for treatment decisions are not usually captured in databases that can be examined retrospectively. Such research would be an important first step toward improving NCCN Guideline adherence and evaluating the potential utility of additional supportive care guidelines.

\section{Acknowledgments}

Medical writing support was provided by Micah Robinson at Amgen Inc.

\section{References}

1. Gradishar WJ, Anderson BO, Balassanian R, et al. NCCN Clinical Practice Guidelines in Oncology: Breast Cancer. Version 3.2014. Available at: NCCN.org. Accessed December 9, 2014.

2. Morgan RJ Jr, Armstrong DK, Alvarez RD, et al. NCCN Clinical Practice Guidelines in Oncology: Ovarian Cancer. Version 3.2014. Available at: NCCN.org. Accessed December 9, 2014.

3. Ettinger DS, Wood DE, Akerley W, et al. NCCN Clinical Practice Guidelines in Oncology: Non-Small Cell Lung Cancer. Version 1.2015. Available at: NCCN.org. Accessed December 9, 2014

4. Hoppe RT, Advani RH, Ai WZ, et al. NCCN Clinical Practice Guidelines in Oncology: Hodgkin Lymphoma. Version 2.2014. Available at: NCCN org. Accessed December 9, 2014.

5. Zelenetz AD, Gordon LI, Wierda WG, et al. NCCN Clinical Practice Guidelines in Oncology: Non-Hodgkin's Lymphomas. Version 5.2014. Available at: NCCN.org. Accessed December 9, 2014.

6. Benson AB III, Venook AP, Bekaii-Saab T, et al. NCCN Clinical Practice Guidelines in Oncology: Colon Cancer. Version 2.2015. Available at: NCCN.org. Accessed December 9, 2014.

7. Benson AB III, Venook AP, Bekaii-Saab T, et al. NCCN Clinical Practice Guidelines in Oncology: Rectal Cancer. Version 2.2015. Available at: NCCN.org. Accessed December 9, 2014.

8. Lyman GH, Dale DC, Crawford J. Incidence and predictors of low doseintensity in adjuvant breast cancer chemotherapy: a nationwide study of community practices. J Clin Oncol 2003;21:4524-4531.

9. Crawford J, Dale DC, Kuderer NM, et al. Risk and timing of neutropenic events in adult cancer patients receiving chemotherapy: the results of a prospective nationwide study of oncology practice. J Natl Compr Canc Netw 2008;6:109-118.

10. Lyman GH, Dale DC, Friedberg J, et al. Incidence and predictors of low chemotherapy dose-intensity in aggressive non-Hodgkin's lymphoma: a nationwide study. J Clin Oncol 2004;22:4302-4311.

11. Pettengell $R$, Schwenkglenks $M$, Leonard $R$, et al. Neutropenia occurrence and predictors of reduced chemotherapy delivery: results from the INCEU prospective observational European neutropenia study. Support Care Cancer 2008;16:1299-1309.

12. Bonadonna G, Valagussa $P$, Moliterni A, et al. Adjuvant cyclophosphamide, methotrexate, and fluorouracil in node-positive breast cancer: the results of 20 years of follow-up. N Engl J Med 1995;332:901-906.

13. Bosly A, Bron D, Van Hoof A, et al. Achievement of optimal average relative dose intensity and correlation with survival in diffuse large B-cell lymphoma patients treated with CHOP. Ann Hematol 2008;87:277-283.

14. Chirivella I, Bermejo B, Insa A, et al. Optimal delivery of anthracyclinebased chemotherapy in the adjuvant setting improves outcome of breast cancer patients. Breast Cancer Res Treat 2009;114:479-484.

15. Kwak LW, Halpern J, Olshen RA, Horning SJ. Prognostic significance of actual dose intensity in diffuse large-cell lymphoma: results of a treestructured survival analysis. J Clin Oncol 1990;8:963-977. 
Chemotherapy Relative Dose Intensity

16. Osby E, Hagberg H, Kvaloy $\mathrm{S}$, et al. CHOP is superior to CNOP in elderly patients with aggressive lymphoma while outcome is unaffected by filgrastim treatment: results of a Nordic Lymphoma Group randomized trial. Blood 2003;101:3840-3848.

17. Wildiers H, Reiser M. Relative dose intensity of chemotherapy and its impact on outcomes in patients with early breast cancer or aggressive lymphoma. Crit Rev Oncol Hematol 2011;77:221-240.

18. Havrilesky LJ, Hanna RK, Poniewierski MS, et al. Relationship between relative dose intensity and mortality in women receiving combination chemotherapy for stage III-IV epithelial ovarian cancer [abstract]. J Clin Oncol 2011;29(Suppl):Abstract 5084.

19. Hanna RK, Poniewierski MS, Laskey RA, et al. Predictors of reduced relative dose intensity and its relationship to mortality in women receiving multi-agent chemotherapy for epithelial ovarian cancer. Gynecol Oncol 2013;129:74-80.

20. Lyman GH. Impact of chemotherapy dose intensity on cancer patient outcomes. J Natl Compr Canc Netw 2009;7:99-108.

21. Budman DR, Berry DA, Cirrincione CT, et al. Dose and dose intensity as determinants of outcome in the adjuvant treatment of breast cancer. The Cancer and Leukemia Group B. J Natl Cancer Inst 1998;90:1205-1211.

22. Lyman GH, Dale DC, Tomita D, et al. A retrospective evaluation of chemotherapy dose intensity and supportive care for early-stage breast cancer in a curative setting. Breast Cancer Res Treat 2013;139:863-872.

23. Hryniuk WM, Figueredo A, Goodyear M. Applications of dose intensity to problems in chemotherapy of breast and colorectal cancer. Semin Oncol 1987;14(4 Suppl 4):3-11.

24. Foote M. The Importance of planned dose of chemotherapy on time: do we need to change our clinical practice? Oncologist 1998;3:365-368.

25. Balducci L, Al-Halawani $\mathrm{H}$, Charu V, et al. Elderly cancer patients receiving chemotherapy benefit from first-cycle pegfilgrastim. Oncologist 2007;12:1416-1424

26. Kuderer NM, Dale DC, Crawford J, Lyman GH. Impact of primary prophylaxis with granulocyte colony-stimulating factor on febrile neutropenia and mortality in adult cancer patients receiving chemotherapy: a systematic review. J Clin Oncol 2007;25:3158-3167.

27. Lyman GH, Crawford J, Tomita D, et al. Changing patterns of chemotherapy relative dose intensity and supportive care for aggressive B-cell non-Hodgkin's lymphoma. Leuk Lymphoma 2015:1-8.

28. Weycker D, Barron R, Edelsberg J, et al. Incidence of reduced chemotherapy relative dose intensity among women with early stage breast cancer in US clinical practice. Breast Cancer Res Treat 2012;133:301-310.

29. Crawford J, Becker PS, Armitage JO, et al. NCCN Clinical Practice Guidelines in Oncology: Myeloid Growth Factors. Version 2.2014. Available at: NCCN.org. Accessed December 9, 2014.

30. Hurria A, Wong FL, Villaluna D, et al. Role of age and health in treatment recommendations for older adults with breast cancer: the perspective of oncologists and primary care providers. J Clin Oncol 2008;26:5386-5392.

31. Kornblith AB, Kemeny M, Peterson BL, et al. Survey of oncologists' perceptions of barriers to accrual of older patients with breast carcinoma to clinical trials. Cancer 2002;95:989-996.

32. Citron ML, Berry DA, Cirrincione C, et al. Randomized trial of dosedense versus conventionally scheduled and sequential versus concurrent combination chemotherapy as postoperative adjuvant treatment of nodepositive primary breast cancer: first report of Intergroup Trial C9741/ Cancer and Leukemia Group B Trial 9741. J Clin Oncol 2003;21:14311439.
33. Hurria A, Hurria A, Brogan K, et al. Effect of creatinine clearance on patterns of toxicity in older patients receiving adjuvant chemotherapy for breast cancer. Drugs Aging 2005;22:785-791.

34. Repetto L. Greater risks of chemotherapy toxicity in elderly patients with cancer. J Support Oncol 2003;1:18-24.

35. Giordano SH, Hortobagyi GN, Kau SW, et al. Breast cancer treatment guidelines in older women. J Clin Oncol 2005;23:783-791.

36. van de Water W, Markopoulos C, van de Velde CJ, et al. Association between age at diagnosis and disease-specific mortality among postmenopausal women with hormone receptor-positive breast cancer. JAMA 2012;307:590-597.

37. Griggs JJ, Mangu PB, Anderson H, et al. Appropriate chemotherapy dosing for obese adult patients with cancer: American Society of Clinical Oncology clinical practice guideline. J Clin Oncol 2012;30:1553-1561.

38. Lyman GH, Sparreboom A. Chemotherapy dosing in overweight and obese patients with cancer. Nat Rev Clin Oncol 2013;10:451-459.

39. Muss HB, Woolf S, Berry D, et al. Adjuvant chemotherapy in older and younger women with lymph node-positive breast cancer. JAMA 2005;293:1073-1081.

40. Shayne M, Culakova E, Wolff D, et al. Dose intensity and hematologic toxicity in older breast cancer patients receiving systemic chemotherapy. Cancer 2009; 115:5319-5328

41. Gajra A, Klepin HD, Feng T, et al. Predictors of chemotherapy dose reduction at first cycle in patients age 65 years and older with solid tumors. J Geriatr Oncol 2015;6:133-140.

42. Greer JA, Pirl WF, Park ER, et al. Behavioral and psychological predictors of chemotherapy adherence in patients with advanced non-small cell lung cancer. J Psychosom Res 2008;65:549-552.

43. Repetto L, CIPOMO Investigators. Incidence and clinical impact of chemotherapy induced myelotoxicity in cancer patients: an observational retrospective survey. Crit Rev Oncol Hematol 2009;72:170-179.

44. Elting LS, Rubenstein EB, Martin CG, et al. Incidence, cost, and outcomes of bleeding and chemotherapy dose modification among solid tumor patients with chemotherapy-induced thrombocytopenia. J Clin Oncol 2001;19:1137-1146.

45. Morrow GR, Shelke AR, Roscoe JA, et al. Management of cancer-related fatigue. Cancer Invest 2005;23:229-239.

46. Mucke M, Mochamat, Cuhls $\mathrm{H}$, et al. Pharmacological treatments for fatigue associated with palliative care. Cochrane Database Syst Rev 2015;5:CD006788.

47. Basch E, Prestrud AA, Hesketh PJ, et al. Antiemetics: American Society of Clinical Oncology clinical practice guideline update. J Clin Oncol 2011;29:4189-4198.

48. Speck RM, Sammel MD, Farrar JT, et al. Impact of chemotherapy-induced peripheral neuropathy on treatment delivery in nonmetastatic breast cancer. J Oncol Pract 2013;9:e234-240.

49. Lalla RV, Bowen J, Barasch A, et al. MASCC/ISOO clinical practice guidelines for the management of mucositis secondary to cancer therapy. Cancer 2014;120:1453-1461.

50. Culakova E, Thota R, Poniewierski MS, et al. Patterns of chemotherapyassociated toxicity and supportive care in US oncology practice: a nationwide prospective cohort study. Cancer Med 2014;3:434-444.

51. Giordano SH, Lin YL, Kuo YF, et al. Decline in the use of anthracyclines for breast cancer. J Clin Oncol 2012;30:2232-2239. 
Supplemental online content for:

\section{Dose Delays, Dose Reductions, and}

\section{Relative Dose Intensity in Patients With Cancer Who}

Received Adjuvant or Neoadjuvant Chemotherapy in

\section{Community Oncology Practices}

Neelima Denduluri, MD; Debra A. Patt, MD; Yunfei Wang, PhD; Menaka Bhor, PhD;

Xiaoyan Li, PhD; Anne M. Favret, MD; Phuong Khanh Morrow, MD; Richard L. Barron, MS;

Lina Asmar, PhD; Shanmugapriya Saravanan, MS; Yanli Li, MD, PhD; Jacob Garcia, MD; and Gary H. Lyman, MD, MPH

J Natl Compr Canc Netw 2015;13:xxx-xxx

- eFigure 1: Distribution of Relative Dose Intensity by Standard Tumor Regimen Cohort

- eTable 1: Standard Dose of Myelosuppressive Agents, Number of Cycles, and Cycle Length for Standard Tumor Regimens 

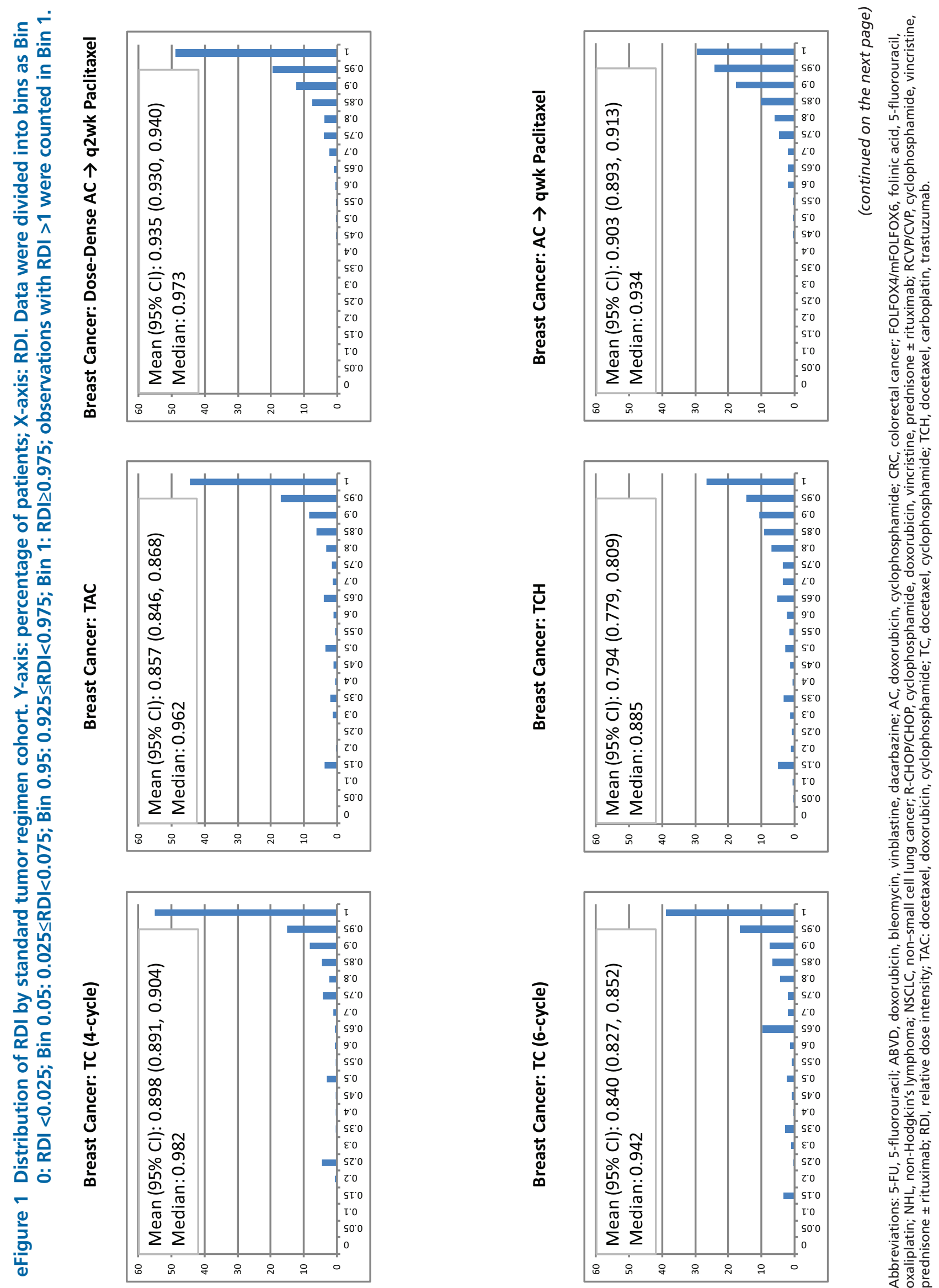

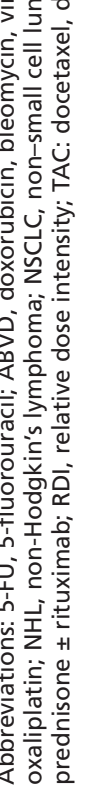



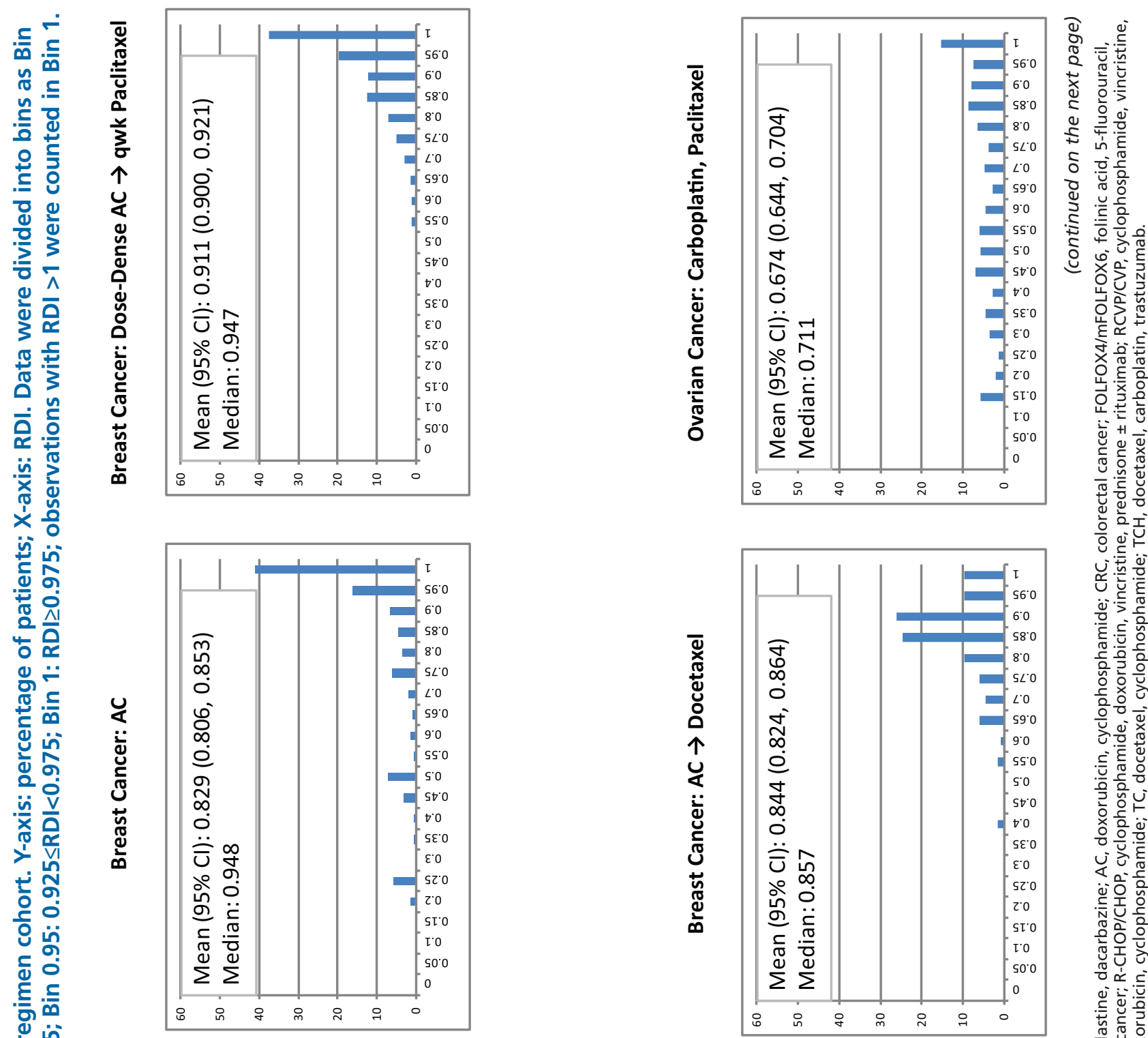

흥 혼

is

농

은 심

产 只

ปั

욜.

ข้ ம்

을

ํํำ

শั

>

ํํํ ช

응

ธ

हू

ข

흥

हํํำ
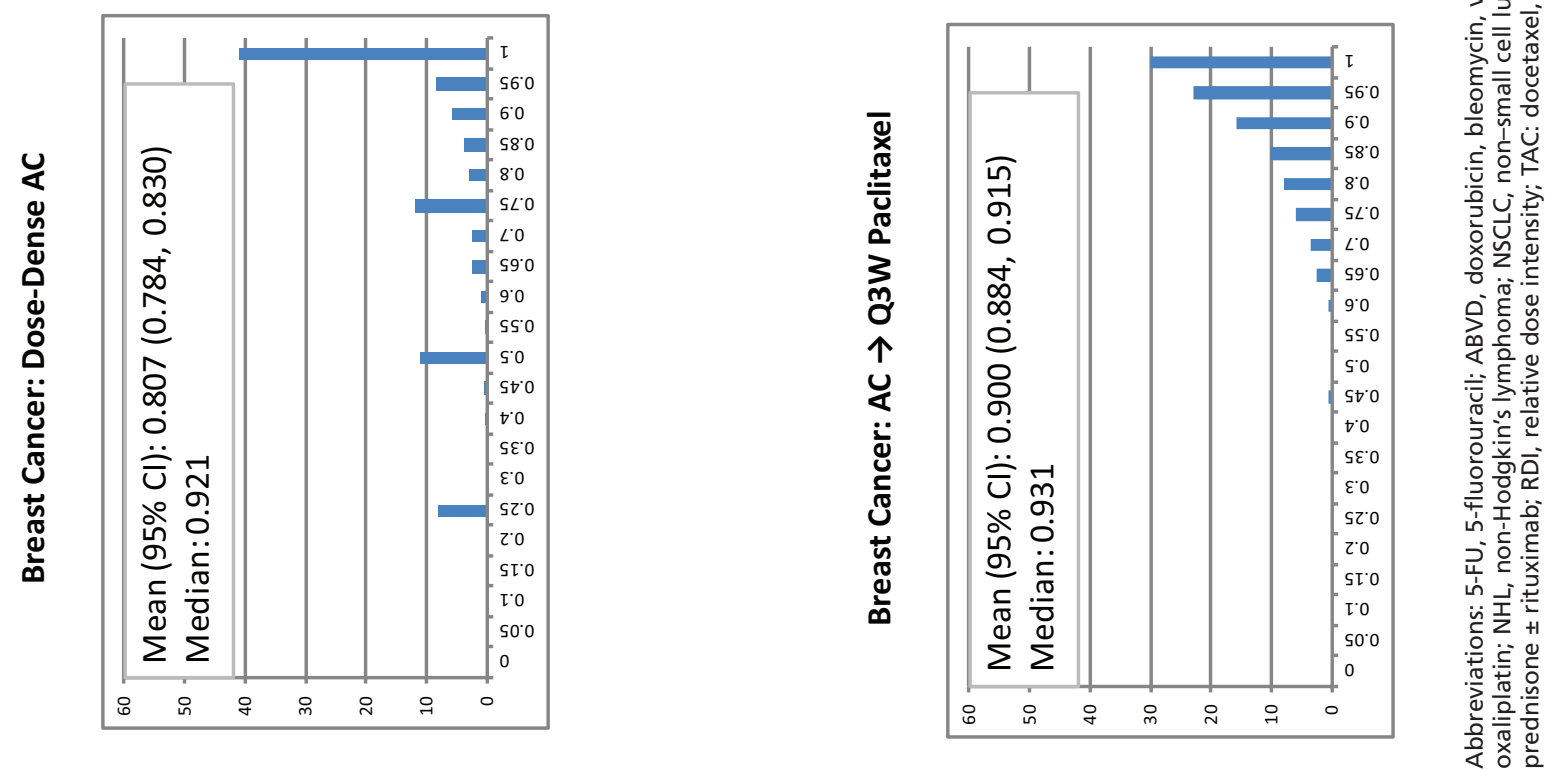

(C) JNCCN_Journal of the National Comprehensive Cancer Network | Volume 13 Number 11 | November 2015 

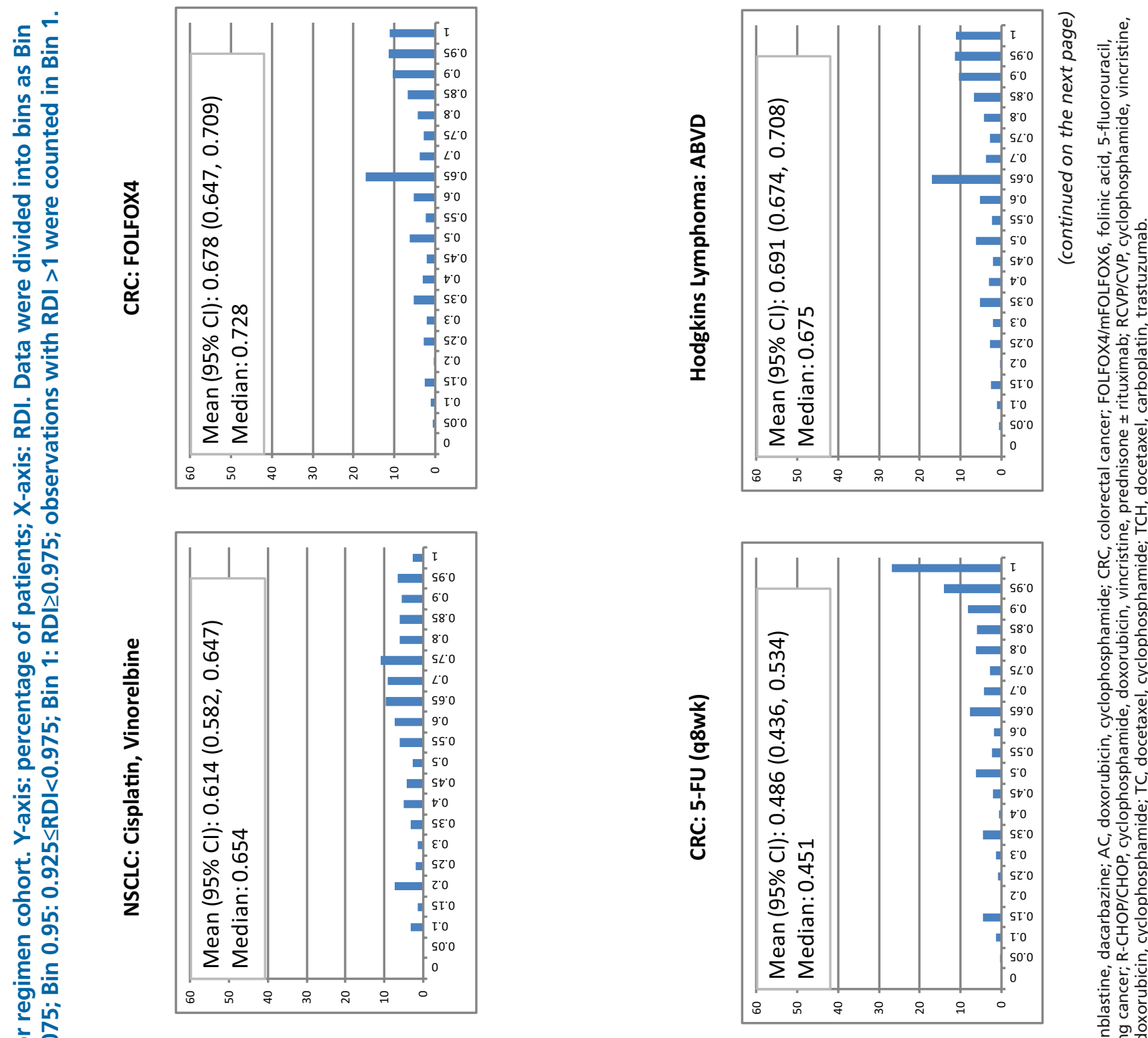

흥

톨인

음

융 능

出

ลำ่

승
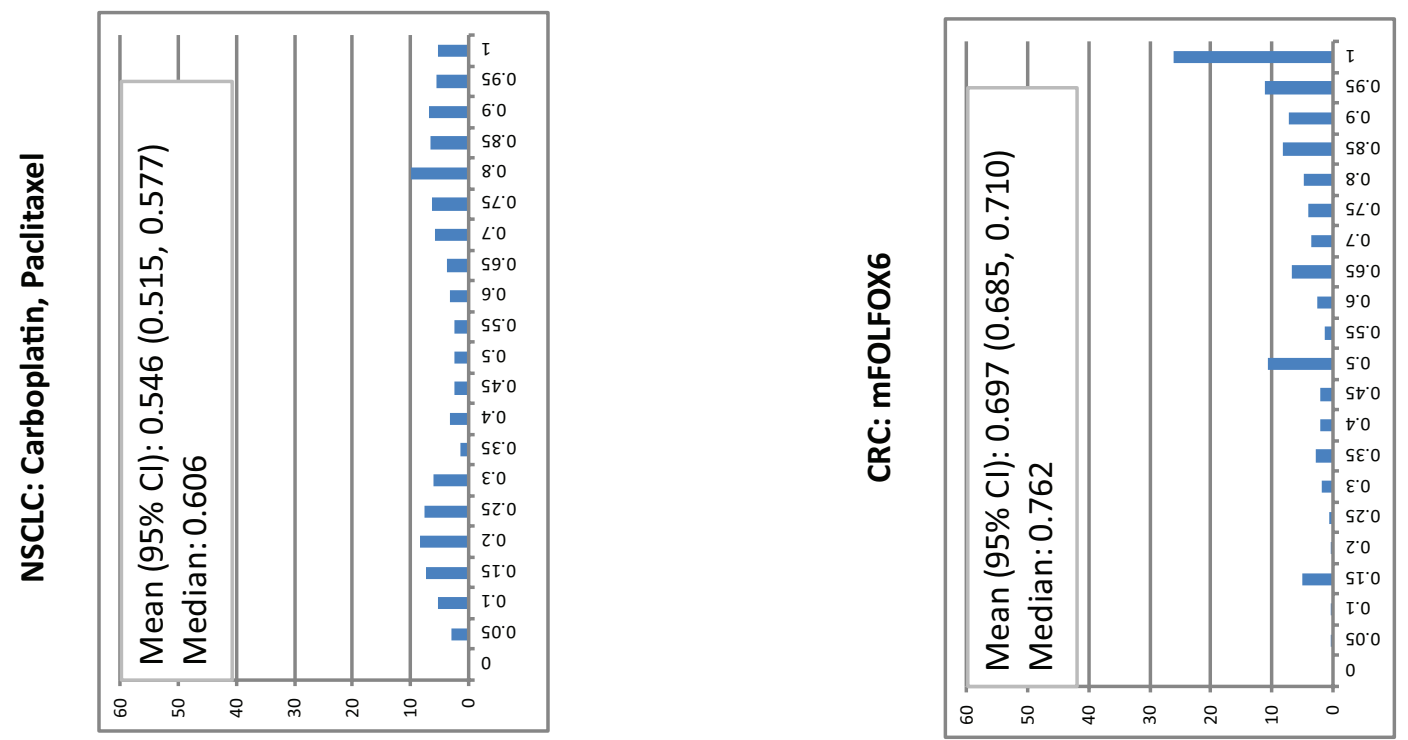

뒥 웓음

可㐫

हिं

政

吾

항

응 ${ }^{2}$

今

孚产잉

言

言.

흑흥 웅

फำ 웅

วิ

它主

塎

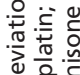



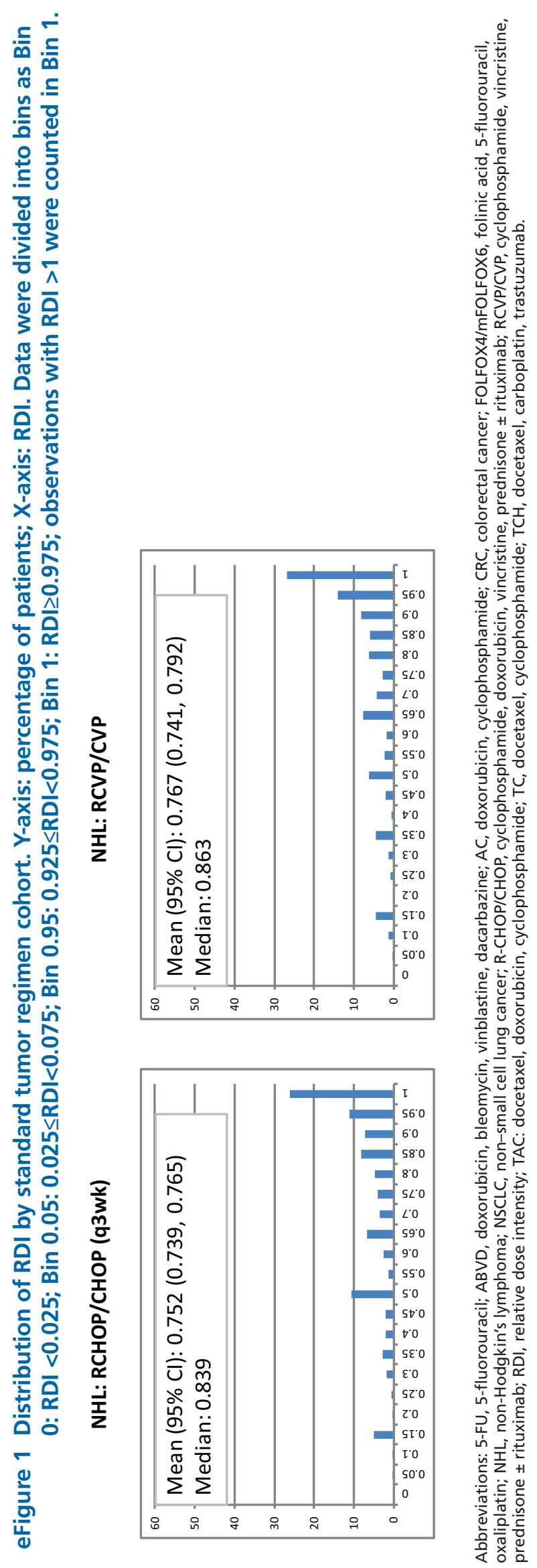

(C) JNCCN_Journal of the National Comprehensive Cancer Network | Volume 13 Number 11 | November 2015 


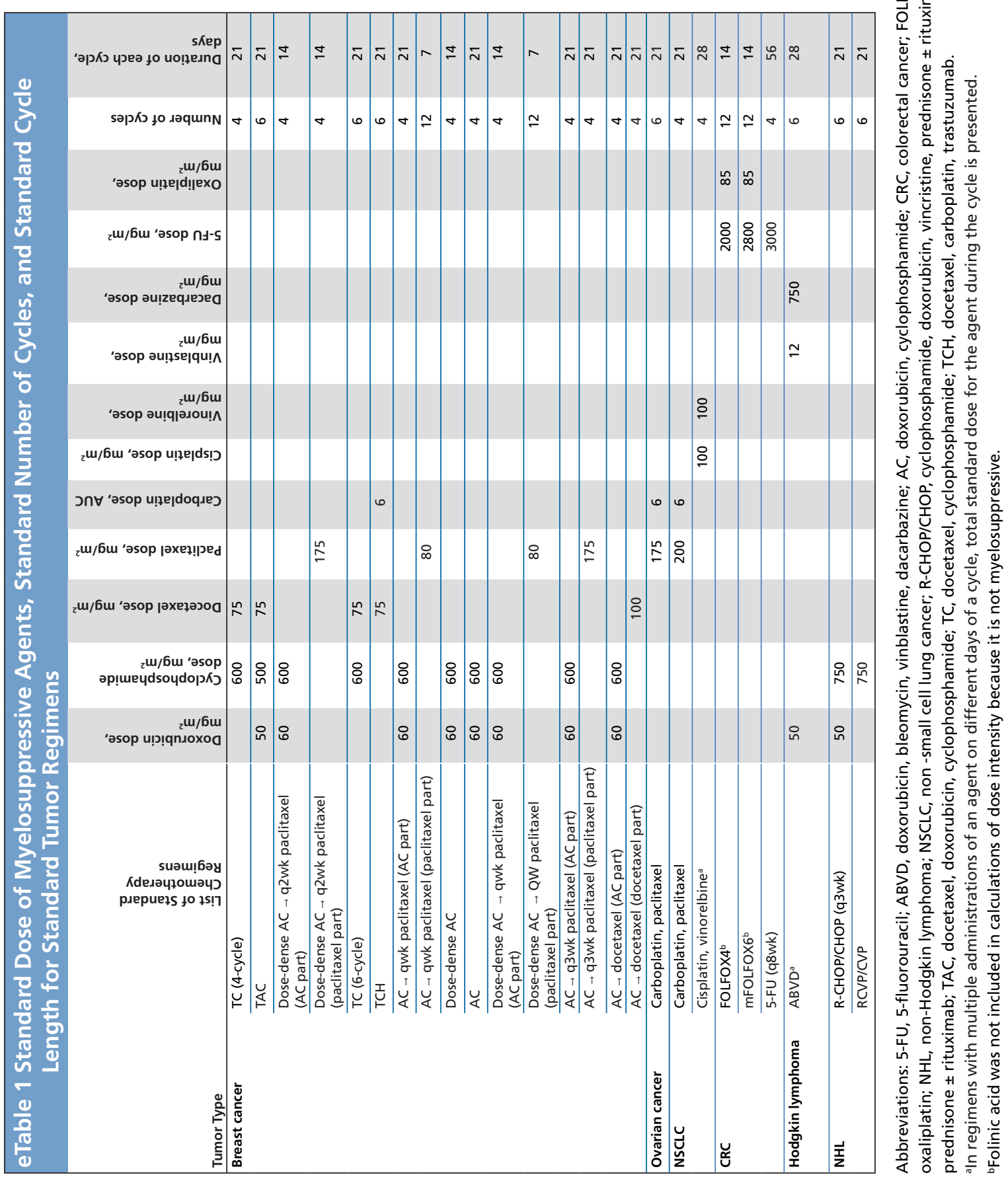

